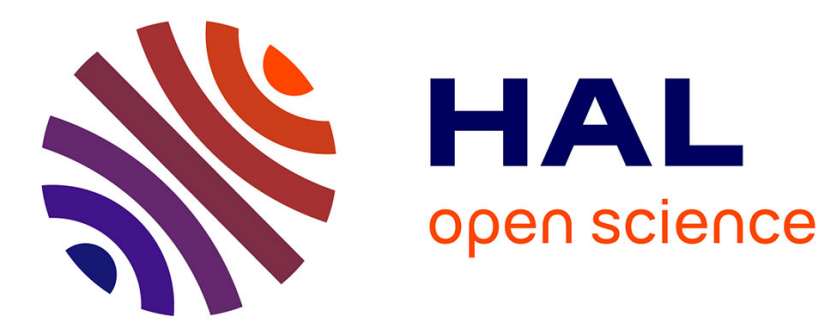

\title{
HIGH-ORDER FULLY WELL-BALANCED LAGRANGE-PROJECTION SCHEME FOR SHALLOW WATER
}

Tomás de Luna, Manuel Jesús Díaz, Christophe Chalons

\section{- To cite this version:}

Tomás de Luna, Manuel Jesús Díaz, Christophe Chalons. HIGH-ORDER FULLY WELLBALANCED LAGRANGE-PROJECTION SCHEME FOR SHALLOW WATER. Communications in Mathematical Sciences, 2020, 18 (3), pp.781-807. 10.4310/CMS.2020.v18.n3.a9 . hal-03993053

\section{HAL Id: hal-03993053 https://hal.science/hal-03993053}

Submitted on 12 Apr 2023

HAL is a multi-disciplinary open access archive for the deposit and dissemination of scientific research documents, whether they are published or not. The documents may come from teaching and research institutions in France or abroad, or from public or private research centers.
L'archive ouverte pluridisciplinaire HAL, est destinée au dépôt et à la diffusion de documents scientifiques de niveau recherche, publiés ou non, émanant des établissements d'enseignement et de recherche français ou étrangers, des laboratoires publics ou privés. 


\title{
High-order fully well-balanced Lagrange-Projection scheme for shallow water
}

\author{
Tomás Morales de Luna* Manuel J. Castro Díaz ${ }^{\dagger} \quad$ Christophe Chalons ${ }^{\ddagger}$
}

June 17, 2019

\begin{abstract}
In this work we propose a novel strategy to define high-order fully well-balanced LagrangeProjection finite volume solvers for balance laws. In particular, we focus on the $1 \mathrm{D}$ shallow water system as it is a reference system of balance laws with non-trivial stationary solutions. Nevertheless, the strategy proposed here could be extended to other interesting balance laws. By fully well-balanced, it is meant that the scheme is able to preserve stationary smooth solutions. Following [6], we exploit the idea of using a high-order well-balanced reconstruction operator for the Lagrangian step. Nevertheless, this is not enough to achieve well-balanced high-order during the projection step. We propose here a new projection step that overcomes this difficulty and that reduces to the standard one in case of conservation laws. Finally, some numerical experiments illustrate the good behaviour of the scheme.
\end{abstract}

\section{Introduction}

Basics. The goal of this paper is to design high-order fully well-balanced finite volume LagrangeProjection numerical schemes for balance laws. In particular we focus on the shallow water system. As usual in Lagrange-Projection schemes, we use the standard Lagrange-Projection decomposition to naturally decouple the acoustic and transport phenomena. We refer for instance the reader to $[10,11,12,13,9,15,16]$ for various applications of such a strategy. It is the purpose of this contribution to set the basis of an extension to higher order accuracy, preserving all (or a representative set of) stationary solutions, which is non trivial due to the Lagrange-Projection decomposition.

As pointed before, in the present work, we will be especially interested in the numerical approximation of the solutions of shallow-water like systems, whose prototype in Eulerian coordinates is given by

$$
\left\{\begin{array}{l}
\partial_{t} h+\partial_{x}(h u)=0 \\
\partial_{t}(h u)+\partial_{x}\left(h u^{2}+g \frac{h^{2}}{2}\right)=-g h \partial_{x} z
\end{array}\right.
$$

where $z(x)$ denotes a given smooth topography, $g>0$ is the gravity constant, while the water depth $h \geq 0$ and velocity $u$ depend on the space and time variables, namely $x \in \mathbb{R}$ and $t \in[0, \infty)$, and are the so-called primitive variables. However, there would be no difficulty to apply a similar approach as the one proposed here to more general systems than (1.1), including for instance sediment transport bedload and suspension, turbidity currents and other geophysical systems like the ones studied in $[17,14,24,22]$. For the sake of conciseness, (1.1) will be given the following condensed form

$$
\partial_{t} \mathbf{U}+\partial_{x} \mathbf{F}(\mathbf{U})=\mathbf{S}(\mathbf{U}) \partial_{x} z,
$$

*Dpto. de Matemáticas. Universidad de Córdoba. Campus de Rabanales. 14071 Córdoba, Spain (tomas.morales@ uco.es).

†Departamento de Análisis Matemático, Facultad de Ciencias, Universidad de Málaga, 29071 Málaga, Spain (mjcastro@uma.es).

${ }^{\ddagger}$ Laboratoire de Mathématiques de Versailles, UVSQ, CNRS, Université Paris-Saclay, 78035 Versailles, France (christophe.chalons@uvsq.fr). 
where $\mathbf{U}=(h, h u)^{T}, \mathbf{F}(\mathbf{U})=\left(h u, h u^{2}+g h^{2} / 2\right)^{T}$ and $\mathbf{S}(\mathbf{U})=(0,-g h)^{T}$. We assume that the initial water depth $h(x, t=0)=h_{0}(x)$ and velocity $u(x, t=0)=u_{0}(x)$ are given at time $t=0$. At last, recall that the left-hand side of (1.1) is strictly hyperbolic over the phase space $\Omega=\left\{(h, h u)^{T} \in \mathbb{R}^{2} \mid h>0\right\}$ with two genuinely non linear characteristic fields associated with the eigenvalues $\{u-c, u+c\}$ where the sound speed is defined by $c=\sqrt{g h}$.

In addition to the high-order accuracy, we expect our scheme to satisfy the now very well-known (fully) well-balanced property. It is meant that discrete approximations of the smooth stationary solutions of (1.1), governed by the ordinary differential system $\partial_{x} F(\mathbf{U})=\mathbf{S}(\mathbf{U}) \partial_{x} z$ or equivalently

$$
h u=\text { constant }, \quad \frac{u^{2}}{2}+g(h+z)=\text { constant }
$$

should be preserved exactly. The particular "lake at rest" stationary solution corresponds to

$$
h+z=\text { constant }, \quad u=0 .
$$

Note that most of the schemes proposed in the literature are focused on these particular stationary solutions and they are said to be well-balanced. We refer for instance the reader to the recent book [19] for a review on the design of well-balanced schemes. However, some numerical schemes also preserve equilibria (1.3) with a non-zero velocity, leading to the so-called fully well-balanced property. We refer for instance the reader to $[23,7,4,25,28,2]$. The objective here is to extend to high-order the recent contribution [9] where we propose a first-order fully well-balanced Lagrange-Projection scheme.

The shallow-water equations in Lagrangian coordinates. As already said, we aim at proposing a high-order and fully well-balanced numerical scheme based on a Lagrange-Projection decomposition. Therefore, the so-called Lagrangian coordinates which amount to describe the flow by following the fluid motion, will play an important role. With this in mind, and for any given "fluid particle" $\xi$, we introduce the characteristic curves

$$
\left\{\begin{array}{l}
\frac{\partial x}{\partial t}(\xi, t)=u(x(\xi, t), t) \\
x(\xi, 0)=\xi
\end{array}\right.
$$

and given any function $(x, t) \rightarrow \mathbf{U}(x, t)$ in Eulerian coordinates, we denote by

$$
\overline{\mathbf{U}}(\xi, t)=\mathbf{U}(x(\xi, t), t)
$$

its counterpart in Lagrangian coordinates $(\xi, t)$. Note that since we aim at following every particle $\xi$, the new functions $\overline{\mathbf{U}}$ in Lagrangian coordinates depend only on the time $t$ for all $\xi$. To go further, note that if we define

$$
L(\xi, t)=\frac{\partial x}{\partial \xi}(\xi, t)
$$

which satisfies

$$
\left\{\begin{array}{l}
\frac{\partial L}{\partial t}(\xi, t)=\partial_{\xi} u(x(\xi, t), t) \\
L(\xi, 0)=1
\end{array}\right.
$$

we also have

$$
\partial_{t} L(\xi, t)=\partial_{\xi} u(x(\xi, t), t)=\partial_{\xi} \bar{u}(\xi, t)
$$

and for all $\mathbf{U}$

$$
\partial_{\xi} \overline{\mathbf{U}}(\xi, t)=L(\xi, t) \partial_{x} \mathbf{U}(x, t) \quad \text { and } \quad \partial_{t} \overline{\mathbf{U}}(\xi, t)=\partial_{t} \mathbf{U}(x, t)+u(x, t) \partial_{x} \mathbf{U}(x, t) .
$$

Therefore and since the shallow water equations (1.1) in Eulerian coordinates are equivalent for smooth solutions to

$$
\left\{\begin{array}{l}
\partial_{t} h+u \partial_{x} h+h \partial_{x} u=0 \\
\partial_{t}(h u)+u \partial_{x}(h u)+h u \partial_{x} u+\partial_{x} p+g h \partial_{x} z=0
\end{array}\right.
$$


where we have set $p=g h^{2} / 2$, we get by multiplying the two equations by $L(\xi, t)$ that

$$
\left\{\begin{array}{l}
L(\xi, t) \partial_{t} \bar{h}(\xi, t)+\bar{h} \partial_{\xi} \bar{u}(\xi, t)=0 \\
L(\xi, t) \partial_{t} \overline{(h u)}(\xi, t)+\overline{h u} \partial_{\xi} \bar{u}+\partial_{\xi} \bar{p}+g \bar{h} \partial_{\xi} \bar{z}=0
\end{array}\right.
$$

and finally, since $\partial_{t} L(\xi, t)=\partial_{\xi} \bar{u}(\xi, t)$ (see above),

$$
\left\{\begin{array}{l}
\partial_{t}(L \bar{h})=0 \\
\partial_{t}(L \overline{h u})+\partial_{\xi} \bar{p}+g \bar{h} \partial_{\xi} \bar{z}=0 .
\end{array}\right.
$$

The functions now depend on $(\xi, t)$ in $(1.11)$, instead on $(x, t)$ in $(1.1)$.

The Lagrange-Projection algorithm. The proposed algorithm based on a Lagrange-Projection decomposition to solve (1.1) will simply consist in first solving system (1.11) in Lagrangian coordinates, and then to come back to the Eulerian coordinates by a projection step. The details will be given hereafter. The extension to high-order of this two-step algorithm is not straightforward, more so if we want to preserve all smooth stationary solutions as well. The major difficulty comes from the fact that the stationary solutions in the Lagrangian framework depend on time. In order to obtain a fully well-balanced Lagrange-Projection scheme, we exploit the idea of using a high-order wellbalanced reconstruction operator for the Lagrangian step. Nevertheless, this is not enough to achieve well-balanced high-order during the projection step. We propose here a new projection step that overcomes this difficulty and takes into account that stationary solutions depend on time.

Outline of the paper. The paper is organized in such a way that the high-order and well-balanced properties are treated separately. More precisely, one first considers in the next section the case of a flat topography, which makes the source term in (1.1) trivial $\left(\partial_{x} z=0\right)$, and focus ourselves on the high-order extension of the Lagrange-Projection scheme. Then, we show in Section 3 how to deal with the general case of a non constant topography and satisfy at the same time the high-order accuracy and fully well-balanced properties. At last, Section 4 collects some numerical results in order to illustrate the behavior of the proposed strategies.

\section{The case of a flat topography}

In this section, the topography is taken to be flat, leading to the classical shallow water system over flat bottom or, in a more general framework the barotropic gas dynamics equations, in Eulerian coordinates:

$$
\left\{\begin{array}{l}
\partial_{t} h+\partial_{x}(h u)=0 \\
\partial_{t}(h u)+\partial_{x}\left(h u^{2}\right)+\partial_{x} p=0
\end{array}\right.
$$

and in Lagrangian coordinates:

$$
\left\{\begin{array}{l}
\partial_{t}(L \bar{h})=0 \\
\partial_{t}(L \overline{h u})+\partial_{\xi} \bar{p}=0
\end{array}\right.
$$

In order to approximate the solutions of this system, space and time will be discretized using a space step $\Delta x$ and a time step $\Delta t$ into a set of cells $\left[x_{i-1 / 2}, x_{i+1 / 2}\right)$ and instants $t^{n}=n \Delta t$, where $x_{i+1 / 2}=i \Delta x$ and $x_{i}=\left(x_{i-1 / 2}+x_{i+1 / 2}\right) / 2$ are respectively the cell interfaces and cell centers, for $i \in \mathbb{Z}$ and $n \in \mathbb{N}$. For a given initial condition $x \mapsto \mathbf{U}^{0}(x)$ where $\mathbf{U}=(h, h u)^{t}$, we will consider a discrete initial data $\mathbf{U}_{i}^{0}$ which approximates $\frac{1}{\Delta x} \int_{x_{i-1 / 2}}^{x_{i+1 / 2}} \mathbf{U}^{0}(x) \mathrm{d} x$, for $i \in \mathbb{Z}$. Therefore, the proposed algorithm aims at computing an approximation $\mathbf{U}_{i}^{n}$ of $\frac{1}{\Delta x} \int_{x_{i-1 / 2}}^{x_{i+1 / 2}} \mathbf{U}\left(x, t^{n}\right) \mathrm{d} x$ where $x \rightarrow \mathbf{U}\left(x, t^{n}\right)$ is the exact solution of the shallow water equations at all time $t^{n}, n \in \mathbb{N}$. Given the sequence $\left\{\mathbf{U}_{i}^{n}\right\}_{i \in \mathbb{Z}}$, it is a matter of defining the sequence $\left\{\mathbf{U}_{i}^{n+1}\right\}_{i \in \mathbb{Z}}, n \in \mathbb{N}$, since $\left\{\mathbf{U}_{i}^{0}\right\}_{i \in \mathbb{Z}}$ is assumed to be known.

Using these notations, the overall Lagrange-Projection algorithm can be described as follows: for a given discrete state $\mathbf{U}_{i}^{n}=(h, h u)_{i}^{n}, i \in \mathbb{Z}$ that describes the system at instant $t^{n}$, the computation of the approximation $\mathbf{U}_{i}^{n+1}=(h, h u)_{i}^{n+1}$ at the next time level is a two-step process defined by 
1. Update $\mathbf{U}_{i}^{n}$ to $\overline{\mathbf{U}}_{i}^{n+1}$ by approximating the solution of (1.11) in Lagrangian coordinates, which is referred to as the Lagrangian step,

2. Update $\overline{\mathbf{U}}_{i}^{n+1}$ to $\mathbf{U}_{i}^{n+1}$ by going back to the Eulerian coordinates, referred to as the Projection (or Transport) step.

In this section, we aim at proposing a high-order (in space and time) approximation of these two steps, in such a way that the overall algorithm is high-order too. To do so, we will focus first on the high-order approximation in space, so that the time is left continuous and a semi-discrete in time scheme shall be proposed. Afterwards, an explicit Runge-Kutta scheme will be applied to obtain a high-order in time scheme.

\subsection{The Lagrangian step}

First of all, we consider for the $\xi$ variable the same space discretization as for the $x$ variable, that is to say $\Delta \xi=\Delta x, \xi_{i+1 / 2}=x_{i+1 / 2}$ and $\xi_{i}=x_{i}$ for all $i$. Therefore, it is natural to approximate (2.2) by the semi-discrete in time scheme

$$
\left\{\begin{array}{l}
\frac{d}{d t}(L \bar{h})_{i}(t)=0 \\
\frac{d}{d t}(L \overline{h u})_{i}(t)=-\frac{1}{\Delta x}\left(\pi_{i+1 / 2}^{*}(t)-\pi_{i-1 / 2}^{*}(t)\right)
\end{array}\right.
$$

where

$$
\pi_{i \pm 1 / 2}^{*}(t) \approx p\left(\xi_{j \pm 1 / 2}, t\right)
$$

and with, according to (1.8),

$$
L_{i}(0)=1
$$

and

$$
L_{i}(t)=\frac{1}{\Delta x}\left(\int_{x_{i-1 / 2}}^{x_{i+1 / 2}} \frac{\partial x}{\partial \xi}(\xi, t) d \xi\right)=\frac{x_{i+1 / 2}^{*}(t)-x_{i-1 / 2}^{*}(t)}{\Delta x},
$$

where $x_{i \pm 1 / 2}^{*}(t)$ naturally represents the position at time $t$ of the exact trajectory defined by the ordinary differential equation

$$
\left\{\begin{array}{l}
\frac{\partial x}{\partial t}\left(\xi_{i \pm 1 / 2}, t\right)=u\left(x\left(\xi_{i \pm 1 / 2}, t\right), t\right) \\
x\left(\xi_{i \pm 1 / 2}, 0\right)=\xi_{i \pm 1 / 2}
\end{array}\right.
$$

that is

$$
x_{i \pm 1 / 2}^{*}=x\left(\xi_{i \pm 1 / 2}, t\right) .
$$

In what follows and for the sake of simplicity we shall not write the dependence on time whenever there is no ambiguity. As usual in finite volume methods, $\pi_{i \pm 1 / 2}^{*}$ and $u_{i \pm 1 / 2}^{*}$ will be classically defined thanks to two given two-point numerical flux functions $u^{*}(.,$.$) and \pi^{*}(.,$.$) that we assume to be consistent,$ namely $u^{*}(\mathbf{U}, \mathbf{U})=u(\mathbf{U})$ and $\pi^{*}(\mathbf{U}, \mathbf{U})=p(\mathbf{U})$ for all $\mathbf{U}$. In practice, the definition of these functions will be based on the one given in $[18,10,13,9]$ evaluated at the inter-cell reconstructed values as will be described in the next subsection.

\subsubsection{High-order approximation in space}

In order to construct a high-order approximation in space, we make use of a reconstruction operator of order $s$ that associates to a given sequence $\left\{\mathbf{U}_{i}\right\}_{i \in \mathbb{Z}}$ two new sequences $\left\{\mathbf{U}_{i+1 / 2+}\right\}_{i \in \mathbb{Z}}$ and $\left\{\mathbf{U}_{i+1 / 2-}\right\}_{i \in \mathbb{Z}}$ such that whenever

$$
\mathbf{U}_{i}(t)=\frac{1}{\Delta x} \int_{x_{i-1 / 2}}^{x_{i+1 / 2}} \mathbf{U}(x, t) d x
$$


for some smooth vector function $x \rightarrow \mathbf{U}(x, t)$, then

$$
\mathbf{U}_{i+1 / 2 \pm}=\mathbf{U}\left(x_{i+1 / 2}, t\right)+\mathcal{O}\left(\Delta x^{s}\right)
$$

for all $i$. In practice, $\mathbf{U}_{i+1 / 2 \pm}$ are computed as follows:

$$
\mathbf{U}_{i-1 / 2+}(t)=\lim _{x \rightarrow x_{i-1 / 2}} \mathbf{P}_{i}^{t}(x) \quad \text { and } \quad \mathbf{U}_{i+1 / 2-}(t)=\lim _{x \rightarrow x_{i+1 / 2}} \mathbf{P}_{i}^{t}(x),
$$

where $x \rightarrow \mathbf{P}_{i}^{t}(x)$ represents a vector of polynomials reconstructed on the cell $\left[x_{i-1 / 2}, x_{i+1 / 2}\right)$ using for instance ENO or WENO techniques, see [27]. These polynomials are expected to be conservative in the sense that for all $i$

$$
\frac{1}{\Delta x} \int_{x_{i-1 / 2}}^{x_{i+1 / 2}} \mathbf{P}_{i}^{t}(x) d x=\mathbf{U}_{i}(t) .
$$

In a very classical way, we thus set

$$
u_{i+1 / 2}^{*}=u^{*}\left(\mathbf{U}_{i+1 / 2-}, \mathbf{U}_{i+1 / 2+}\right), \quad \pi_{i+1 / 2}^{*}=\pi^{*}\left(\mathbf{U}_{i+1 / 2-}, \mathbf{U}_{i+1 / 2+}\right)
$$

for all $i$. More explicitly, following [18, 10, 12, 9], we set

$$
\begin{gathered}
u_{j+1 / 2}^{*}=u^{*}\left(\mathbf{U}_{j+1 / 2-}, \mathbf{U}_{j+1 / 2+}\right):=\frac{1}{2}\left(u_{j+1 / 2-}+u_{j+1 / 2+}\right)-\frac{1}{2 a_{j+1 / 2}}\left(p_{j+1 / 2+}-p_{j+1 / 2-}\right), \\
\pi_{j+1 / 2}^{*}=\pi^{*}\left(\mathbf{U}_{j+1 / 2-}, \mathbf{U}_{j+1 / 2+}\right):=\frac{1}{2}\left(p_{j+1 / 2-}+p_{j+1 / 2+}\right)-\frac{a_{j+1 / 2}}{2}\left(u_{j+1 / 2+}-u_{j+1 / 2-}\right),
\end{gathered}
$$

and $p_{j+1 / 2 \pm}=g\left(h_{j+1 / 2 \pm}\right)^{2} / 2$ for all $j$.

Here, the constant $a_{j+1 / 2}$ has to be chosen sufficiently large for the sake of stability, and more precisely larger than the Lagrangian sound speed according to the well-known subcharacteristic condition. In practice it is required that $a_{i+1 / 2}$ is greater than the values $h \sqrt{g h}$ at the interface:

$$
a_{j+1 / 2}=\max \left\{h_{i+1 / 2-} \sqrt{g h_{j+1 / 2-}}, h_{i+1 / 2+} \sqrt{g h_{j+1 / 2+}}\right\} .
$$

In other words, the treatment of the Lagrange step exactly fits within the framework of standard high-order finite volume schemes.

\subsection{The Projection step}

In order to project the piece-wise constant approximate values of $L \overline{\mathbf{U}}(\xi, t), \mathbf{U}=(h, h u)^{t}$, obtained on each cell $\left(\xi_{i-1 / 2}, \xi_{i+1 / 2}\right)$ at the end of the first step, it is now a matter of defining a high-order approximation of $\mathbf{U}(x, t)$, on the Eulerian cells $\left(x_{i-1 / 2}, x_{i+1 / 2}\right)$. With this in mind, we first notice the (trivial but) key property

$$
\int_{x\left(\xi_{l}, t\right)}^{x\left(\xi_{r}, t\right)} \mathbf{U}(x, t) d x=\int_{\xi_{l}}^{\xi_{r}} L(\xi, t) \overline{\mathbf{U}}(\xi, t) d \xi .
$$

Given a time $t \geq 0$ we define $\hat{\xi}_{i+1 / 2}(t)$ such that

$$
x\left(\hat{\xi}_{i+1 / 2}(t), t\right)=x_{i+1 / 2} .
$$

Thus, for any time $T \geq 0, \hat{\xi}(T)$ corresponds to the origin of the characteristic $x\left(\hat{\xi}_{i+1 / 2}, t\right)$ such that at time $t=T$ coincides with $x_{i+1 / 2}$ (see Figure 1 ).

Remark that, given a fixed time $T \geq 0$, we have for any $t \geq 0$

$$
\left\{\begin{array}{l}
\frac{\partial x}{\partial t}\left(\hat{\xi}_{i+1 / 2}(T), t\right)=u\left(x\left(\hat{\xi}_{i+1 / 2}(T), t\right), t\right) \\
x\left(\hat{\xi}_{i+1 / 2}(T), 0\right)=\hat{\xi}_{i+1 / 2}(T)
\end{array}\right.
$$




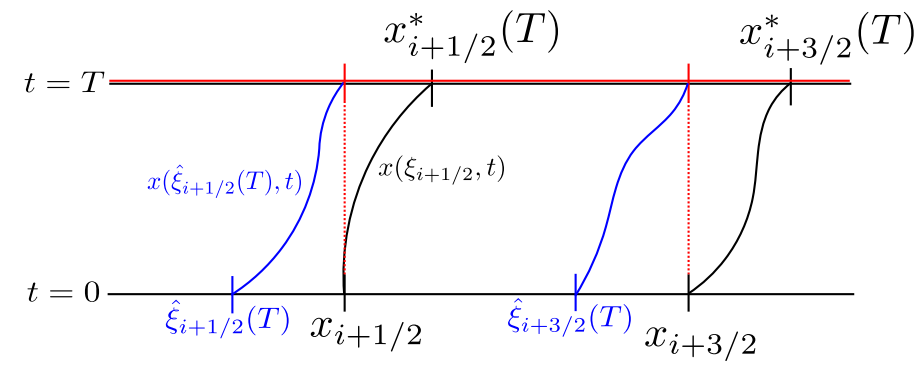

Figure 1: Sketch of the connection between Lagrangian and Eulerian coordinates

which we may approximate (at first-order in time) by

$$
x_{i+1 / 2}=x\left(\hat{\xi}_{i+1 / 2}(T), T\right) \approx x\left(\hat{\xi}_{i+1 / 2}(T), 0\right)+T \frac{\partial x}{\partial t}\left(\hat{\xi}_{i+1 / 2}(T), 0\right) \approx \hat{\xi}_{i+1 / 2}+T u_{i+1 / 2}^{*}
$$

Then, we naturally set for $\mathbf{U}=(h, h u)^{t}$,

$$
\mathbf{U}_{i}(t)=\frac{1}{\Delta x} \int_{x_{i-1 / 2}}^{x_{i+1 / 2}} \mathbf{U}(x, t) d x=\frac{1}{\Delta x} \int_{x\left(\hat{\xi}_{i-1 / 2}(t), t\right)}^{x\left(\hat{\xi}_{i+1 / 2}(t), t\right)} \mathbf{U}(x, t) d x=\frac{1}{\Delta x} \int_{\hat{\xi}_{i-1 / 2}(t)}^{\hat{\xi}_{i+1 / 2}(t)} L(\xi, t) \overline{\mathbf{U}}(\xi, t) d \xi
$$

and we split the integral as follows

$$
\begin{aligned}
\mathbf{U}_{i}(t)=\frac{1}{\Delta x} \int_{\hat{\xi}_{i-1 / 2}(t)}^{\xi_{i-1 / 2}} L(\xi, t) \overline{\mathbf{U}}(\xi, t) d \xi+ & \\
& +\frac{1}{\Delta x} \int_{\xi_{i-1 / 2}}^{\xi_{i+1 / 2}} L(\xi, t) \overline{\mathbf{U}}(\xi, t) d \xi+\frac{1}{\Delta x} \int_{\xi_{i+1 / 2}}^{\hat{\xi}_{i+1 / 2}(t)} L(\xi, t) \overline{\mathbf{U}}(\xi, t) d \xi
\end{aligned}
$$

Note that the middle integral in the right-hand-side naturally equals $(L \overline{\mathbf{U}})_{i}(t)$ and this quantity is known from the first step. It remains to evaluate the other two integrals at high-order accuracy in space.

\subsubsection{First-order approximation}

As a preliminary scheme, it is interesting to note that at first-order accuracy in the time interval $\left[t^{n}, t^{n+1}\right]$, writing the dependence on $t^{n}$ or $t^{n+1}$ as a superscript $(.)^{n}$ or $(.)^{n+1}$ respectively, we may set

$$
(L \overline{\mathbf{U}})_{i-1 / 2}^{n+1}= \begin{cases}(L \overline{\mathbf{U}})_{i-1}^{n+1}, & \text { for } \xi_{i-1 / 2}>\hat{\xi}_{i-1 / 2}, \\ (L \overline{\mathbf{U}})_{i}^{n+1}, & \text { for } \xi_{i-1 / 2} \leq \hat{\xi}_{i-1 / 2}\end{cases}
$$

or equivalently

$$
(L \overline{\mathbf{U}})_{i-1 / 2}^{n+1}= \begin{cases}(L \overline{\mathbf{U}})_{i-1}^{n+1}, & \text { for } x_{i-1 / 2}^{*, n+1}>x_{i-1 / 2}, \\ (L \overline{\mathbf{U}})_{i}^{n+1}, & \text { for } x_{i-1 / 2}^{*, n+1} \leq x_{i-1 / 2},\end{cases}
$$

where, we are considering

$$
\begin{aligned}
& x_{i+1 / 2}^{*, n+1}=x_{i+1 / 2}+\Delta t u_{i+1 / 2}^{*}, \\
& x_{i+1 / 2}=\hat{\xi}_{i+1 / 2}+\Delta t u_{i+1 / 2}^{*} .
\end{aligned}
$$


We are led to set

$$
\frac{1}{\Delta x} \int_{\hat{\xi}_{i-1 / 2}}^{\xi_{i-1 / 2}} L(\xi, \Delta t) \overline{\mathbf{U}}^{n+1}(\xi) d \xi=\frac{\xi_{i-1 / 2}-\hat{\xi}_{i-1 / 2}}{\Delta x}(L \overline{\mathbf{U}})_{i-1 / 2}^{n+1}
$$

and similarly

$$
\frac{1}{\Delta x} \int_{\xi_{i+1 / 2}}^{\hat{\xi}_{i+1 / 2}} L(\xi, \Delta t) \overline{\mathbf{U}}^{n+1}(\xi) d \xi=\frac{\hat{\xi}_{i+1 / 2}-\xi_{i+1 / 2}}{\Delta x}(L \overline{\mathbf{U}})_{i+1 / 2}^{n+1}
$$

Therefore, we get

$$
\begin{gathered}
\mathbf{U}_{i}^{n+1}=\frac{\xi_{i-1 / 2}-\hat{\xi}_{i-1 / 2}}{\Delta x}(L \overline{\mathbf{U}})_{i-1 / 2}^{n+1}+(L \overline{\mathbf{U}})_{i}^{n+1}+\frac{\hat{\xi}_{i+1 / 2}-\xi_{i+1 / 2}}{\Delta x}(L \overline{\mathbf{U}})_{i+1 / 2}^{n+1}= \\
=(L \overline{\mathbf{U}})_{i}^{n+1}-\frac{\Delta t}{\Delta x}\left(u_{i+1 / 2}^{*, n}(L \overline{\mathbf{U}})_{i+1 / 2}^{n+1}-u_{i-1 / 2}^{*, n}(L \overline{\mathbf{U}})_{i-1 / 2}^{n+1}\right)
\end{gathered}
$$

where

$$
(L \overline{\mathbf{U}})_{i-1 / 2}^{n+1}= \begin{cases}(L \overline{\mathbf{U}})_{i-1}^{n+1}, & \text { for } x_{i-1 / 2}^{*, n+1}>x_{i-1 / 2} \\ (L \overline{\mathbf{U}})_{i}^{n+1}, & \text { for } x_{i-1 / 2}^{*, n+1} \leq x_{i-1 / 2}\end{cases}
$$

As we will see in the next subsection, this approach is especially well-adapted to be extended to high-order accuracy, although it does not correspond to the "usual" first-order Projection step as underlined in the following remark.

Remark. The "usual" Projection step, see for instance [9], amounts to define

$$
\overline{\mathbf{U}}_{i-1 / 2}^{n+1}= \begin{cases}\overline{\mathbf{U}}_{i-1}^{n+1}, & \text { for } \xi_{i-1 / 2}>\hat{\xi}_{i-1 / 2}, \\ \overline{\mathbf{U}}_{i}^{n+1}, & \text { for } \xi_{i-1 / 2} \leq \hat{\xi}_{i-1 / 2},\end{cases}
$$

or equivalently

$$
\overline{\mathbf{U}}_{i-1 / 2}^{n+1}= \begin{cases}\overline{\mathbf{U}}_{i-1}^{n+1}, & \text { for } x_{i-1 / 2}^{*, n+1}>x_{i-1 / 2}, \\ \overline{\mathbf{U}}_{i}^{n+1}, & \text { for } x_{i-1 / 2}^{*, n+1} \leq x_{i-1 / 2}\end{cases}
$$

and to set, first

$$
\begin{gathered}
\frac{1}{\Delta x} \int_{\hat{\xi}_{i-1 / 2}}^{\xi_{i-1 / 2}} L(\xi, \Delta t) \overline{\mathbf{U}}^{n+1}(\xi) d \xi=\overline{\mathbf{U}}_{i-1 / 2}^{n+1} \frac{1}{\Delta x} \int_{\hat{\xi}_{i-1 / 2}}^{\xi_{i-1 / 2}} L(\xi, \Delta t) d \xi=\overline{\mathbf{U}}_{i-1 / 2}^{n+1} \frac{1}{\Delta x} \int_{\hat{\xi}_{i-1 / 2}}^{\xi_{i-1 / 2}} \partial_{\xi} x(\xi, \Delta t) d \xi \\
=\overline{\mathbf{U}}_{i-1 / 2}^{n+1} \frac{x\left(\xi_{i-1 / 2}, \Delta t\right)-x\left(\hat{\xi}_{i-1 / 2}, \Delta t\right)}{\Delta x}=\frac{x_{i-1 / 2}^{*, n+1}-x_{i-1 / 2}}{\Delta x} \overline{\mathbf{U}}_{i-1 / 2}^{n+1},
\end{gathered}
$$

and second (in a similar way)

$$
\begin{gathered}
\frac{1}{\Delta x} \int_{\xi_{i+1 / 2}}^{\hat{\xi}_{i+1 / 2}} L(\xi, \Delta t) \overline{\mathbf{U}}^{n+1}(\xi) d \xi=\overline{\mathbf{U}}_{i+1 / 2}^{n+1} \frac{1}{\Delta x} \int_{\xi_{i+1 / 2}}^{\hat{\xi}_{i+1 / 2}} L(\xi, \Delta t) d \xi=\overline{\mathbf{U}}_{i+1 / 2}^{n+1} \frac{1}{\Delta x} \int_{\xi_{i+1 / 2}}^{\hat{\xi}_{i+1 / 2}} \partial_{\xi} x(\xi, \Delta t) d \xi \\
=\overline{\mathbf{U}}_{i+1 / 2}^{n+1} \frac{x\left(\hat{\xi}_{i+1 / 2}, \Delta t\right)-x\left(\xi_{i+1 / 2}, \Delta t\right)}{\Delta x}=\frac{x_{i+1 / 2}-x_{i+1 / 2}^{*, n+1}}{\Delta x} \overline{\mathbf{U}}_{i+1 / 2}^{n+1} .
\end{gathered}
$$

Therefore, we get

$$
\begin{array}{r}
\mathbf{U}_{i}^{n+1}=\frac{x_{i-1 / 2}^{*, n+1}-x_{i-1 / 2}}{\Delta x} \overline{\mathbf{U}}_{i-1 / 2}^{n+1}+\frac{x_{i+1 / 2}^{*, n+1}-x_{i-1 / 2}^{*, n+1}}{\Delta x} \overline{\mathbf{U}}_{i}^{n+1}+\frac{x_{i+1 / 2}-x_{i+1 / 2}^{*, n+1}}{\Delta x} \overline{\mathbf{U}}_{i+1 / 2}^{n+1} \\
=(L \overline{\mathbf{U}})_{i}^{n+1}-\frac{\Delta t}{\Delta x}\left(u_{i+1 / 2}^{*, n} \overline{\mathbf{U}}_{i+1 / 2}^{n+1}-u_{i-1 / 2}^{*, n} \overline{\mathbf{U}}_{i-1 / 2}^{n+1}\right),
\end{array}
$$


where

$$
\overline{\mathbf{U}}_{i-1 / 2}^{n+1}= \begin{cases}\overline{\mathbf{U}}_{i-1}^{n+1}, & \text { for } x_{i-1 / 2}^{*, n+1}>x_{i-1 / 2}, \\ \overline{\mathbf{U}}_{i}^{n+1}, & \text { for } x_{i-1 / 2}^{*, n+1} \leq x_{i-1 / 2} .\end{cases}
$$

In the above formulas, $\overline{\mathbf{U}}_{i}^{n+1}$ are naturally defined by $\overline{\mathbf{U}}_{i}^{n+1}=(L \overline{\mathbf{U}})_{i}^{n+1} / L_{i}^{n+1}$. This classical approach may be not suitable to the high-order extension because dividing by $L_{i}^{n+1} \approx 1+\Delta t\left(\partial_{\xi} \bar{u}\right)_{i}$ could introduce a first-order error with respect to time.

\subsubsection{High-order approximation in space}

In order to construct a high-order approximation in space, we proceed as in the first step by making use of a similar polynomial reconstruction operator of order $s$ but now related to the piecewise average values $L \overline{\mathbf{U}}_{i}(t)$. Again, we impose the conservation property, that is to say if we denote $\xi \rightarrow L \mathbf{P}_{i}^{t}(\xi)$ the corresponding polynomial on the $i^{t h}$-cell,

$$
\int_{\xi_{i-1 / 2}}^{\xi_{i+1 / 2}} L \mathbf{P}_{i}^{t}(\xi) d \xi=L \overline{\mathbf{U}}_{i}(t)
$$

As for the first-order accuracy, it is a matter of defining the three integrals in the right-hand side of

$$
\begin{aligned}
\mathbf{U}_{i}(t)=\frac{1}{\Delta x} \int_{\hat{\xi}_{i-1 / 2}(t)}^{\xi_{i-1 / 2}} L(\xi, t) \overline{\mathbf{U}}(\xi, t) d \xi & \\
& +\frac{1}{\Delta x} \int_{\xi_{i-1 / 2}}^{\xi_{i+1 / 2}} L(\xi, t) \overline{\mathbf{U}}(\xi, t) d \xi+\frac{1}{\Delta x} \int_{\xi_{i+1 / 2}}^{\hat{\xi}_{i+1 / 2}(t)} L(\xi, t) \overline{\mathbf{U}}(\xi, t) d \xi .
\end{aligned}
$$

Thanks to the conservation property of the reconstruction, the middle integral of the right-hand-side naturally equals $(L \overline{\mathbf{U}})_{i}(t)$. To evaluate the first and third integrals, we simply suggest to use the polynomial reconstruction of $L \mathbf{P}_{i}^{t}(\xi, t)$ in the corresponding cell, that is to say, if we consider for instance the first integral

$$
\frac{1}{\Delta x} \int_{\hat{\xi}_{i-1 / 2}(t)}^{\xi_{i-1 / 2}} L(\xi, t) \overline{\mathbf{U}}(\xi, t) d \xi
$$

we will consider the polynomial $\xi \rightarrow L \mathbf{P}_{i-1}^{t}(\xi)$ of the cell $i-1$ if $\xi_{i-1 / 2}>\hat{\xi}_{i-1 / 2}(t)$ and the polynomial $\xi \rightarrow L \mathbf{P}_{i}^{t}(\xi)$ of the cell $i$ otherwise. Then, denoting by $\xi \rightarrow L \mathbf{P}_{i+1 / 2}^{t}(\xi)$ the resulting polynomial we consider a Gauss quadrature formula exact for this polynomial with nodes $\xi_{i+1 / 2, k}$ and weights $\omega_{k}$, for $k=1, \ldots, m$.

The integral

$$
\frac{1}{\Delta x} \int_{\xi_{i+1 / 2}}^{\hat{\xi}_{i+1 / 2}(t)} L(\xi, \Delta t) \overline{\mathbf{U}}(\xi, t) d \xi
$$

is calculated in a similar way and the projection step writes as

$$
\begin{aligned}
\mathbf{U}_{i}(t)=(L \overline{\mathbf{U}})_{i}(t)-\frac{\xi_{i+1 / 2}-\hat{\xi}_{i+1 / 2}}{\Delta x} \sum_{k=1}^{m} \omega_{k} L \mathbf{P}_{i+1 / 2}^{t}\left(\xi_{i+1 / 2, k}\right) & \\
& +\frac{\xi_{i-1 / 2}-\hat{\xi}_{i-1 / 2}}{\Delta x} \sum_{k=1}^{m} \omega_{k} L \mathbf{P}_{i-1 / 2}^{t}\left(\xi_{i-1 / 2, k}\right) .
\end{aligned}
$$

Using $u_{i \pm 1 / 2}^{*}$ to integrate the characteristic backwards, we could replace $\xi_{i \pm 1 / 2}-\hat{\xi}_{i \pm 1 / 2}$ by $x_{i \pm 1 / 2}^{*}-$ $x_{i \pm 1 / 2}$. 


\subsection{High-order integration in time}

So far, we focused ourselves on the high-order accuracy in space. We now briefly discuss the highorder accuracy in time, that we propose to deal with classical explicit Runge-Kutta TVD techniques, see $[26,20]$. However, two crucial remarks are in order. Firstly, the time integration of the exact trajectories (1.8) to define $x\left(\hat{\xi}_{i+1 / 2}, t^{n}\right)$ and $L_{i}^{n+1}$ in the Lagrange and Projection steps must be treated using high-order Runge-Kutta TVD techniques

Secondly, the main (additional) high-order Runge-Kutta TVD scheme must be applied to the overall algorithm, that is to say to the two-step process made of the Lagrange and the Projection step, and not to the Lagrange and the Projection step separately to avoid splitting errors.

Another way to achieve high-order in time is to consider high-order ADER or Taylor schemes like the one proposed in [15].

\section{The case of a non flat topography and well-balanced prop- erty}

In this section, the topography is not taken to be flat anymore, meaning that we consider the original system (1.1) in Eulerian coordinates, or (1.11) in Lagrangian coordinates. Our objective is to show how to extend the high-order Lagrange-Projection scheme proposed in the previous section to this system, paying a particular attention to the well-balanced property associated with stationary solutions satisfying

$$
\frac{u^{2}}{2}+g(h+z)=\text { constant }, \quad q=\text { constant } .
$$

Remark that this implies that any smooth stationary solution satisfies

$$
\partial_{x}\left(h u^{2}+g \frac{h^{2}}{2}\right)+g h \partial_{x} z=0 .
$$

The main ingredients are the hydrostatic reconstruction originally introduced in $[1,7,9]$, and its extension to high-order accuracy introduced in [5] and based on space reconstructions performed on the so-called fluctuations.

Considering the Lagrangian step, it is natural to replace the update formula (2.3) by

$$
\left\{\begin{array}{l}
\frac{d}{d t}(L \bar{h})_{i}(t)=0 \\
\frac{d}{d t}(L \overline{h u})_{i}(t)=-\frac{1}{\Delta x}\left(\pi_{i+1 / 2}^{*, l}(t)-\pi_{i-1 / 2}^{*, r}(t)\right)-\left\{g \bar{h} \partial_{\xi} \bar{z}\right\}_{i}^{t},
\end{array}\right.
$$

where $\pi_{i+1 / 2}^{*, l}$ and $\pi_{i-1 / 2}^{*, r}$ still approximates the value of pressure at the interfaces, while $\left\{g \bar{h} \partial_{\xi} \bar{z}\right\}_{i}^{t}$ approximates the spatial-average value of the exact source term, namely

$$
\left\{g \bar{h} \partial_{\xi} \bar{z}\right\}_{i}^{t} \approx \frac{1}{\Delta x} \int_{\xi_{i-1 / 2}}^{\xi_{i+1 / 2}} g \bar{h}(\xi, t) \partial_{\xi} \bar{z}(\xi, t) d x
$$

The definition of such a semi-discrete scheme is based on the values $\pi_{i+1 / 2}^{*, l}(t)$ and $\pi_{i-1 / 2}^{*, r}(t)$ and $\left\{g \bar{h} \partial_{\xi} \bar{z}\right\}_{i}^{t}$. In what follows, we shall define these terms based on a well-balanced high-order reconstruction procedure. Remark that, in general, the values $\pi_{i+1 / 2}^{*, l}$ and $\pi_{i-1 / 2}^{*, r}$ should take into account the jump on the interfaces of the source term. Nevertheless, we shall assume here a continuous reconstruction of the bottom topography which allows us to define $\pi_{i+1 / 2}^{*, l}=\pi_{i+1 / 2}^{*, r}=\pi_{i+1 / 2}^{*}$. Once the Lagrangian variables are computed, a projection step similar to the one described for the case of flat bottom will be required. The scheme shall be decomposed into three steps:

1. Well-balanced high-order reconstruction 
2. Lagrangian step

\section{Projection step}

that we now give in details.

\subsection{Well-balanced high-order reconstruction}

In order to define a well-balanced reconstruction strategy at high-order accuracy in space, we follow $[5,6,8]$ and perform a high-order polynomial reconstruction on the so-called fluctuations $\mathbf{D}$ defined hereafter. More precisely, consider the cell averages $\left\{\mathbf{U}_{i}(t)\right\}_{i \in \mathbb{Z}}$ and assume that for each cell $i \in \mathbb{Z}$ we can compute a stationary solution $\mathbf{U}_{i}^{t, e}(x)$ such that

$$
\frac{1}{\Delta x} \int_{x_{i-1 / 2}}^{x_{i+1 / 2}} \mathbf{U}_{i}^{t, e}(x) d x=\mathbf{U}_{i}(t) .
$$

Remark that although the stationary solution itself does not depend on time, the selected stationary solution will not be in general the same. For each time $t$ the given stationary solution depends on the value $\mathbf{U}_{i}(t)$ and therefore varies through time.

Then, for any given $i$, we define the stencil $\{i-l, \ldots, i+r\}$ and for $k=i-l, \ldots, i+r$ the differences

$$
\mathbf{D}_{k, i}(t)=\mathbf{U}_{k}(t)-\frac{1}{\Delta x} \int_{x_{k-1 / 2}}^{x_{k+1 / 2}} \mathbf{U}_{i}^{t, e}(x) d x,
$$

on which we will apply a standard reconstruction operator of order $s$ and denoted

$$
\mathbf{P}_{i}^{t}\left(x ; X_{i-l}, \ldots, X_{i+r}\right)
$$

with the property

$$
\mathbf{P}_{i}^{t}(x ; 0, \ldots, 0)=0 .
$$

For instance, if one considers second-order accuracy and the usual MUSCL [21] reconstruction approach, one is led to consider

$$
\mathbf{P}_{i}^{t}\left(x ; X_{i-1}, X_{i}, X_{i+1}\right)=X_{i}+s_{i} \frac{x-x_{i}}{\Delta x},
$$

with the following definition of the slope $s_{i}$ using the well-known minmod limiter,

$$
s_{i}=\operatorname{minmod}\left(X_{i+1}-X_{i}, X_{i}-X_{i-1}\right) .
$$

Then, we set

$$
\mathbf{P}_{i}^{t}(x)=\mathbf{P}_{i}^{t}\left(x ; \mathbf{D}_{i-l, i}(t), \ldots, \mathbf{D}_{i+r, i}(t)\right), \quad x \in C_{i} .
$$

At last, we define the following reconstruction function of the conservative variable $\mathbf{U}$, namely

$$
\mathbf{U}_{i}^{t}(x)=\mathbf{P}_{i}^{t}(x)+\mathbf{U}_{i}^{t, e}(x), \quad x \in C_{i} .
$$

Note that the function $x \rightarrow \mathbf{U}_{i}^{t}(x)$ is not polynomial, but reads like the sum of a polynomial contribution and a portion of the well-balanced reconstruction curve associated to the cell $C_{i}$, the average of which being equal to $\mathbf{U}_{i}$.

Note that system (3.3) may not have a unique solution:

- If (3.3) has no solution, then $U_{i}^{t, e}(x) \equiv 0$ is chosen in the first stage and the reconstruction operator reduces to the standard one. Notice that the reconstruction operator is still wellbalanced, since (3.3) has always at least one solution when the operator is applied to the cell averages of a stationary solution.

- If (3.3) has more than one solution, a criterion to select one of them is needed: see, for instance, [16]. 


\subsection{The Lagrangian step}

We show in this section how to extend the discretization of the Lagrangian step in order to satisfy both the high-order accuracy and well-balanced properties. Again, we first focus on the space discretization. Therefore, the time is left continuous and we assume as given the cell averages $\left\{\mathbf{U}_{i}^{0}\right\}_{i \in \mathbb{Z}}$ at the initial time $t=t_{0}$, which are reconstructed as described before. In order to make the notation less cumbersome, we shall not write explicitly the dependence of the selected stationary solution $\mathbf{U}_{i}^{e}$ on time. This leads to the function

$$
\mathbf{U}_{i}^{0}(x)=\mathbf{P}_{i}^{0}(x)+\mathbf{U}_{i}^{e}(x), \quad x \in C_{i},
$$

where we have used clear notations between the so-called fluctuation and equilibrium parts. More generally

$$
\mathbf{U}_{i}^{t}(x)=\mathbf{P}_{i}^{t}(x)+\mathbf{U}_{i}^{e}(x), \quad x \in C_{i},
$$

for later times. In the following, we will also use the notation $\mathbf{U}_{i}^{f}(x, t)=\mathbf{P}_{i}^{t}(x)$ for the sake of clarity. Based on these reconstructions and as motivated above, we naturally set

$$
\left\{\begin{array}{l}
\frac{d}{d t}(L \bar{h})_{i}(t)=0 \\
\frac{d}{d t}(L \overline{h u})_{i}(t)=-\frac{1}{\Delta x}\left(\pi_{i+1 / 2}^{*}(t)-\pi_{i-1 / 2}^{*}(t)\right)-\left\{g \bar{h} \partial_{\xi} \bar{z}\right\}_{i}^{t},
\end{array}\right.
$$

with $(L \overline{\mathbf{U}})_{i}\left(t_{0}\right)=\mathbf{U}_{i}^{0}$, and where the interfacial pressures are defined by

$$
\pi_{i+1 / 2}^{*}(t)=\pi^{*}\left(\mathbf{U}_{i+1 / 2-}^{t}, \mathbf{U}_{i+1 / 2+}^{t}\right)
$$

for all $i$ where

$$
\mathbf{U}_{i-1 / 2+}^{t}=\lim _{x \rightarrow x_{i-1 / 2}} \mathbf{U}_{i}^{t}(x), \quad \mathbf{U}_{i+1 / 2-}^{t}=\lim _{x \rightarrow x_{i+1 / 2}} \mathbf{U}_{i}^{t}(x) .
$$

Regarding the source term $\left\{g \bar{h} \partial_{\xi} \bar{z}\right\}_{i}^{t}$ and by linearity with respect to $h$, we use the equilibrium and fluctuation decomposition (3.5) to set

$$
\left\{g \bar{h} \partial_{\xi} \bar{z}\right\}_{i}^{t}=\left\{g \bar{h}^{e} \partial_{\xi} \bar{z}\right\}_{i}^{t}+\left\{g \bar{h}^{f} \partial_{\xi} \bar{z}\right\}_{i}^{t}
$$

where with clear notations

$$
\begin{array}{r}
-\left\{g \bar{h}^{e} \partial_{\xi} \bar{z}\right\}_{i}^{t}=-\frac{1}{\Delta x} \int_{\xi_{i-1 / 2}}^{\xi_{i+1 / 2}} g \bar{h}_{i}^{e}(\xi, t) \partial_{\xi} \bar{z}(\xi, t) d \xi=\frac{1}{\Delta x} \int_{\xi_{i-1 / 2}}^{\xi_{i+1 / 2}} \partial_{\xi}\left(\overline{h u}_{i}^{e} \bar{u}_{i}^{e}+\bar{p}_{i}^{e}\right)(\xi, t) d \xi \\
=\frac{1}{\Delta x} \overline{h u}_{i}^{e}(t)\left(\bar{u}_{i+1 / 2-}^{e}(t)-\bar{u}_{i-1 / 2+}^{e}(t)\right)+\frac{1}{\Delta x}\left(\bar{p}_{i+1 / 2-}^{e}(t)-\bar{p}_{i-1 / 2+}^{e}(t)\right)
\end{array}
$$

with

$$
\bar{p}_{i \pm 1 / 2 \mp}^{e}(t)=p_{i}^{e}\left(x\left(\xi_{i \pm 1 / 2}, t\right), t\right)=g \frac{\left(h_{i}^{e}\left(x\left(\xi_{i \pm 1 / 2}, t\right), t\right)^{2}\right.}{2}
$$

and

$$
\bar{u}_{i \pm 1 / 2 \mp}^{e}(t)=u_{i}^{e}\left(x\left(\xi_{i \pm 1 / 2}, t\right), t\right) .
$$

Finally,

$$
\left\{g \bar{h}^{f} \partial_{\xi} \bar{z}\right\}_{i}^{t}=\frac{1}{\Delta x} \int_{\xi_{i-1 / 2}}^{\xi_{i+1 / 2}} g \bar{h}_{i}^{f}(\xi, t) \partial_{\xi} \bar{z}(\xi, t) d \xi
$$

is computed via a (high-order) Gaussian quadrature formula with nodes $x_{j, k}$ and weights $\omega_{k}$ for $k=1, \ldots, m$, leading to

$$
\left\{g \bar{h}^{f} \partial_{\xi} \bar{z}\right\}_{i}^{t}=g \sum_{k=1}^{m} \omega_{i} \bar{h}_{i}^{f}\left(x_{i, k}, t\right) \partial_{\xi} \bar{z}\left(x_{i, k}\right)
$$




\subsection{The Projection step}

In order to get the high-order accuracy property, the projection step proposed here is similar to the case of flat topography in the sense that it is based on polynomial reconstructions of $L \overline{\mathbf{U}}_{i}(t)$. However, in order to satisfy the well-balanced property we propose a new projection algorithm that exactly preserves the smooth stationary solutions, that, as pointed before, depend on time in the Lagrangian framework. This new projection algorithm exploits again the decomposition into stationary and fluctuation parts as in [5, 8]. More precisely we aim at performing high-order polynomial reconstructions on the fluctuation parts of $L \overline{\mathbf{U}}_{i}(t)$. Before going on, it is therefore a matter of defining the fluctuation and equilibrium parts of $L \overline{\mathbf{U}}_{i}(t)$. With this in mind, we consider that the equilibrium part of $L \overline{\mathbf{U}}_{i}(t)$, that we denote by $L \overline{\mathbf{U}}_{i}^{e}(t)$, is given by nothing but (3.6) applied to the equilibrium solution $\mathbf{U}_{i}^{e}(x)$, namely

$$
\left\{\begin{array}{l}
\frac{d}{d t}\left(L \bar{h}^{e}\right)_{i}(t)=0 \\
\frac{d}{d t}\left(L \overline{h u}^{e}\right)_{i}(t)=-\frac{1}{\Delta x}\left(\bar{p}_{i+1 / 2-}^{e}(t)-\bar{p}_{i-1 / 2+}^{e}(t)\right)-\left\{g \bar{h}^{e} \partial_{\xi} \bar{z}\right\}_{i}^{t},
\end{array}\right.
$$

with $L \overline{\mathbf{U}}_{i}^{e}\left(t_{0}\right)=\mathbf{U}_{i}^{e}$. Defining now the fluctuation part by the consistency relation

$$
L \overline{\mathbf{U}}_{i}^{f}(t)=L \overline{\mathbf{U}}_{i}(t)-L \overline{\mathbf{U}}_{i}^{e}(t)
$$

we notice that

$$
\left\{\begin{array}{l}
\frac{d}{d t}\left(L \bar{h}^{f}\right)_{i}(t)=0 \\
\frac{d}{d t}\left(L \overline{h u}^{f}\right)_{i}(t)=-\frac{1}{\Delta x}\left(\pi_{i+1 / 2}^{*}-\bar{p}_{i+1 / 2-}^{e}-\pi_{i-1 / 2}^{*}+\bar{p}_{i-1 / 2+}^{e}\right)-\left\{g \bar{h}^{f} \partial_{\xi} \bar{z}\right\}_{i}^{t}
\end{array}\right.
$$

with $L \overline{\mathbf{U}}_{i}^{f}\left(t_{0}\right)=\mathbf{P}_{i}^{0}$.

Then, in agreement with section 2.2 , we set

$$
\begin{aligned}
\mathbf{U}_{i}(t)=\frac{1}{\Delta x} \int_{\hat{\xi}_{i-1 / 2}(t)}^{\xi_{i-1 / 2}} L(\xi, t) \overline{\mathbf{U}}(\xi, t) d \xi+ & \\
& +\frac{1}{\Delta x} \int_{\xi_{i-1 / 2}}^{\xi_{i+1 / 2}} L(\xi, t) \overline{\mathbf{U}}(\xi, t) d \xi+\frac{1}{\Delta x} \int_{\xi_{i+1 / 2}}^{\hat{\xi}_{i+1 / 2}(t)} L(\xi, t) \overline{\mathbf{U}}(\xi, t) d \xi
\end{aligned}
$$

which is equivalent to

$$
\begin{aligned}
& \mathbf{U}_{i}(t)=\frac{1}{\Delta x} \int_{\hat{\xi}_{i-1 / 2}(t)}^{\xi_{i-1 / 2}} L(\xi, t) \overline{\mathbf{U}}^{e}(\xi, t) d \xi+ \\
& \quad+\frac{1}{\Delta x} \int_{\xi_{i-1 / 2}}^{\xi_{i+1 / 2}} L(\xi, t) \overline{\mathbf{U}}^{e}(\xi, t) d \xi+\frac{1}{\Delta x} \int_{\xi_{i+1 / 2}}^{\hat{\xi}_{i+1 / 2}(t)} L(\xi, t) \overline{\mathbf{U}}^{e}(\xi, t) d \xi \\
& +\frac{1}{\Delta x} \int_{\hat{\xi}_{i-1 / 2}(t)}^{\xi_{i-1 / 2}} L(\xi, t) \overline{\mathbf{U}}^{f}(\xi, t) d \xi+ \\
& +\frac{1}{\Delta x} \int_{\xi_{i-1 / 2}}^{\xi_{i+1 / 2}} L(\xi, t) \overline{\mathbf{U}}^{f}(\xi, t) d \xi+\frac{1}{\Delta x} \int_{\xi_{i+1 / 2}}^{\hat{\xi}_{i+1 / 2}(t)} L(\xi, t) \overline{\mathbf{U}}^{f}(\xi, t) d \xi .
\end{aligned}
$$

Note that again

$$
\frac{1}{\Delta x} \int_{\xi_{i-1 / 2}}^{\xi_{i+1 / 2}} L(\xi, t) \overline{\mathbf{U}}^{f}(\xi, t) d \xi=\left(L \overline{\mathbf{U}}^{f}\right)_{i}(t)
$$


Using now the notation $\xi_{i+1 / 2}^{e}(t)$ such that

$$
x^{e}\left(\xi_{i+1 / 2}^{e}, t\right)=x_{i+1 / 2},
$$

where we have considered the characteristic given by the velocity of the stationary solution

$$
\left\{\begin{array}{l}
\frac{\partial x^{e}}{\partial t}(\xi, t)=u^{e}(x(\xi, t), t), \\
x(\xi, 0)=\xi
\end{array}\right.
$$

and the relation

$$
\begin{aligned}
\mathbf{U}_{i}^{e}=\frac{1}{\Delta x} \int_{x_{i-1 / 2}}^{x_{i+1 / 2}} \mathbf{U}_{i}^{e}(x) d x & =\frac{1}{\Delta x} \int_{\xi_{i-1 / 2}^{e}(t)}^{\xi_{i-1 / 2}} L(\xi, t) \overline{\mathbf{U}}^{e}(\xi, t) d \xi+ \\
+ & \frac{1}{\Delta x} \int_{\xi_{i-1 / 2}}^{\xi_{i+1 / 2}} L(\xi, t) \overline{\mathbf{U}}^{e}(\xi, t) d \xi+\frac{1}{\Delta x} \int_{\xi_{i+1 / 2}}^{\xi_{i+1 / 2}^{e}(t)} L(\xi, t) \overline{\mathbf{U}}^{e}(\xi, t) d \xi
\end{aligned}
$$

we get

$$
\begin{aligned}
\mathbf{U}_{i}(t)=\mathbf{U}_{i}^{e} & +\frac{1}{\Delta x} \int_{\hat{\xi}_{i-1 / 2}(t)}^{\xi_{i-1 / 2}^{e}(t)} L(\xi, t) \overline{\mathbf{U}}^{e}(\xi, t) d \xi-\frac{1}{\Delta x} \int_{\hat{\xi}_{i+1 / 2}(t)}^{\xi_{i+1 / 2}^{e}(t)} L(\xi, t) \overline{\mathbf{U}}^{e}(\xi, t) d \xi \\
& +\left(L \overline{\mathbf{U}}^{f}\right)_{i}(t)+\frac{1}{\Delta x} \int_{\hat{\xi}_{i-1 / 2}(t)}^{\xi_{i-1 / 2}} L(\xi, t) \overline{\mathbf{U}}^{f}(\xi, t) d \xi-\frac{1}{\Delta x} \int_{\hat{\xi}_{i+1 / 2}(t)}^{\xi_{i+1 / 2}} L(\xi, t) \overline{\mathbf{U}}^{f}(\xi, t) d \xi .
\end{aligned}
$$

Now we proceed exactly as in section 2.2.2 and define polynomial reconstructions of $L \overline{\mathbf{P}}_{i}^{\#, t}$ where \# stands for $e$ or $f$ (the equilibrium and fluctuation parts) satisfying as usual the conservation property

$$
\frac{1}{\Delta x} \int_{\xi_{i-1 / 2}}^{\xi_{i+1 / 2}} L \overline{\mathbf{P}}_{i}^{\#, t}(x) d x=L \overline{\mathbf{U}}_{i}^{\#}(t)
$$

and use these polynomials, together with high-order Gauss quadrature formulas, to evaluate the integrals. Of course, we consider the polynomials $\xi \rightarrow L P_{i-1}^{\#, t}(\xi), \xi \rightarrow L P_{i}^{\#, t}(\xi)$, or $\xi \rightarrow L P_{i+1}^{\#, t}(\xi)$ depending on whether $\xi$ is to the left or to the right of the values $\xi_{i \pm 1 / 2}$.

\subsection{Fully well-balanced property}

In this section, we prove that the scheme is fully well-balanced. Therefore, we assume that the initial data is a smooth stationary solution that we denote by $x \rightarrow \mathbf{U}^{e}(x)$ and that for all $i$ the initial values $h_{i}(t=0)$ and $(h u)_{i}(t=0)$ are defined by the cell averages of this initial data.

By definition, the equilibrium and fluctuation decomposition (3.4) gives

$$
\mathbf{P}_{i}^{0}(x)=0 \quad \text { and } \quad \mathbf{U}_{i}^{e}(x)=\mathbf{U}^{e}(x)
$$

for all $i$. Therefore, the well-balanced high-order reconstruction corresponds exactly to the stationary solution and the fluctuation part is 0 . This gives immediately

$$
\begin{gathered}
\mathbf{U}_{i+1 / 2-}(t)=\mathbf{U}_{i+1 / 2+}(t)=\mathbf{U}_{i+1 / 2}^{e}, \\
u_{i+1 / 2}^{*}=u_{i+1 / 2}^{e}, \quad \text { and } \quad \pi_{i+1 / 2}^{*}=p_{i+1 / 2}^{e} .
\end{gathered}
$$

As a consequence, the Lagrangian step gives $\left(L \overline{\mathbf{U}}^{f}\right)_{i}(t)=0$ and $(L \overline{\mathbf{U}})_{i}(t)=\left(L \overline{\mathbf{U}}^{e}\right)_{i}(t)$ for all $t$. Moreover, in this case we clearly have $\hat{\xi}_{i+1 / 2}=\xi_{i+1 / 2}^{e}$ so that from (3.11) it is clear that $\mathbf{U}_{i}(t)=\mathbf{U}_{i}^{e}$ for all $t$, which means that the discrete solution is an equilibrium solution for all times. By continuity, it coincides with the initial condition. 


\subsection{High-order integration in time}

Again, this step is strictly identical to the case of a flat topography and it can be easily proved that it does not affect the well-balanced property.

\subsection{Remarks}

In this section, we give two important remarks. The first one states that for a flat topography, the scheme proposed here is equivalent to the one of the previous section. The second one states that at the first-order accuracy, the scheme is equivalent to the fully well-balanced scheme recently proposed in [9].

Let us first consider a flat topography. In this case, the fluctuation are simply given by

$$
\mathbf{D}_{k, i}(t)=\mathbf{U}_{k}(t)-\mathbf{U}_{i}(t)
$$

so that the reconstruction function of the conservative variable $\mathbf{U}$ is given by

$$
\mathbf{U}_{i}^{t}(x)=\mathbf{P}_{i}^{t}\left(x ; \mathbf{U}_{i-l}(t)-\mathbf{U}_{i}(t), \ldots, \mathbf{U}_{i+r}(t)-\mathbf{U}_{i}(t)\right)+\mathbf{U}_{i}(t) .
$$

Under the additional assumption that the reconstruction operator of order $s$ satisfies the property

$$
\mathbf{P}_{i}^{t}\left(x ; X_{i-l}, \ldots, X_{i+r}\right)=\mathbf{P}_{i}^{t}\left(x ; X_{i-l}-Y, \ldots, X_{i+r}-Y\right)+Y \text { for all } Y,
$$

we get

$$
\mathbf{U}_{i}^{t}(x)=\mathbf{P}_{i}^{t}\left(x ; \mathbf{U}_{i-l}(t), \ldots, \mathbf{U}_{i+r}(t)\right)
$$

which means that a very standard reconstruction process is considered. Then, since $z$ is constant, (3.6) boils down to

$$
\left\{\begin{array}{l}
\frac{d}{d t}(L \bar{h})_{i}(t)=0 \\
\frac{d}{d t}(L \overline{h u})_{i}(t)=-\frac{1}{\Delta x}\left(\pi_{i+1 / 2}^{*}(t)-\pi_{i-1 / 2}^{*}(t)\right)
\end{array}\right.
$$

with initial condition $(L \overline{\mathbf{U}})_{i}\left(t_{0}\right)=\mathbf{U}_{i}^{0}$, which is strictly equivalent to the evolution of the Lagrangian step in the case of a flat topography. As far as the evolution (3.7) of the equilibrium part is concerned, the time derivatives are clearly equal to zero, so that $\left(L \overline{\mathbf{U}}^{e}\right)_{i}(t)=\mathbf{U}_{i}^{0}$ for all times $t$. By definition of the fluctuation part, we deduce that

$$
\left(L \overline{\mathbf{U}}^{f}\right)_{i}(t)=(L \overline{\mathbf{U}})_{i}(t)-\mathbf{U}_{i}^{0} .
$$

Finally, (3.11) gives

$$
\begin{aligned}
\mathbf{U}_{i}(t)=\mathbf{U}_{i}^{0} & \\
& +\left(L \overline{\mathbf{U}}^{f}\right)_{i}(t)+\frac{1}{\Delta x} \int_{\hat{\xi}_{i-1 / 2}(t)}^{\xi_{i-1 / 2}} L(\xi, t) \overline{\mathbf{U}}^{f}(\xi, t) d \xi-\frac{1}{\Delta x} \int_{\hat{\xi}_{i+1 / 2}(t)}^{\xi_{i+1 / 2}} L(\xi, t) \overline{\mathbf{U}}^{f}(\xi, t) d \xi .
\end{aligned}
$$

Invoking (3.14) and again the property of the reconstruction operator (3.12), it is clear that the projection step is equivalent to the one proposed for a flat topography.

Assume now that we consider the first-order version of the scheme. In this case, the fluctuation part at the initial time equals zero (note that $\mathbf{D}_{i, i}=0$ ), and (3.6) and (3.7) are strictly equivalent (same equations and same initial condition) so that the fluctuation part is always equal to zero. In addition, we clearly have $\hat{\xi}_{i-1 / 2}(t)=\xi_{i-1 / 2}^{e}(t)$ so that (3.11) gives

$$
\mathbf{U}_{i}(t)=\mathbf{U}_{i}^{e}=
$$




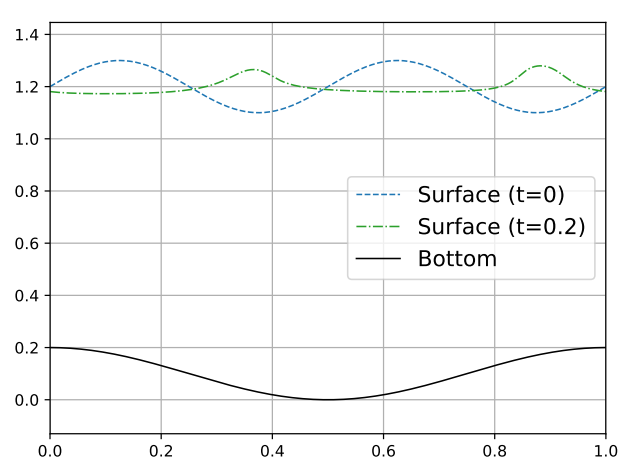

(a) Surface

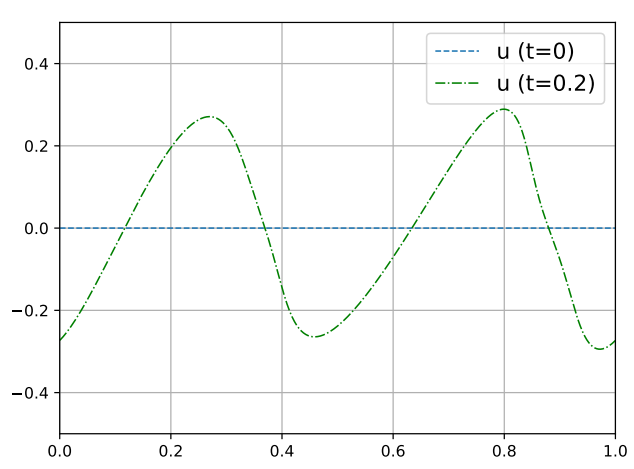

(b) Velocity

Figure 2: Initial and final time evolution

$$
\frac{1}{\Delta x} \int_{\xi_{i-1 / 2}^{e}(t)}^{\xi_{i-1 / 2}} L(\xi, t) \overline{\mathbf{U}}^{e}(\xi, t) d \xi+\frac{1}{\Delta x} \int_{\xi_{i-1 / 2}}^{\xi_{i+1 / 2}} L(\xi, t) \overline{\mathbf{U}}^{e}(\xi, t) d \xi+\frac{1}{\Delta x} \int_{\xi_{i+1 / 2}}^{\xi_{i+1 / 2}^{e}(t)} L(\xi, t) \overline{\mathbf{U}}^{e}(\xi, t) d \xi
$$

and therefore

$$
\begin{gathered}
\mathbf{U}_{i}(t)= \\
\frac{1}{\Delta x} \int_{\hat{\xi}_{i-1 / 2}(t)}^{\xi_{i-1 / 2}} L(\xi, t) \overline{\mathbf{U}}(\xi, t) d \xi+\frac{1}{\Delta x} \int_{\xi_{i-1 / 2}}^{\xi_{i+1 / 2}} L(\xi, t) \overline{\mathbf{U}}(\xi, t) d \xi+\frac{1}{\Delta x} \int_{\xi_{i+1 / 2}}^{\hat{\xi}_{i+1 / 2}(t)} L(\xi, t) \overline{\mathbf{U}}(\xi, t) d \xi .
\end{gathered}
$$

Easy calculations show that this projection step is equivalent to the one proposed in [9] with the choice given in equation (26) of the corresponding section 3.2. Finally, let us remark that we do not discuss here any special treatment for wet/dry fronts or the positivity preserving properties of the scheme and it is out of the scope of this work. Nevertheless, we recall that the first order fully-well balanced Lagrange-Projection [9] is positive preserving and we could combine this scheme together with the strategy proposed by Zhang et al. [29] to obtain a high-order positive preserving scheme.

\section{Numerical experiments}

\subsection{Accuracy test}

We perform first an accuracy test for the scheme. To do so, let us consider in the interval $[0,1]$ a bottom topography given by

$$
z(x)=0.1+0.1 \cos (2 \pi x)
$$

Then consider an initial free surface given by

$$
h(x, t=0)+z(x)=1.1+0.1 \sin (4 \pi x),
$$

and set the velocity initially to $u=0$. We compute a reference solution on a very fine grid with 3200 points up to the time $t=0.2$ with periodic boundary conditions and we compare with the reference solution for different grid meshes using the first, second and third order schemes. Initial and final times are shown in Figure 2 and the comparison for the three schemes with 100 cell points is shown in Figure 3. The $L^{1}$-norm errors are shown in Tables 1, 2 and 3.

\subsection{Perturbation of a lake at rest}

Let us consider a lake at rest initial condition in the interval $[0,1]$ given by

$$
z(x)=0.5 \exp \left(-200(x-0.5)^{2}\right), \quad h+z=1.0, \quad u=0,
$$




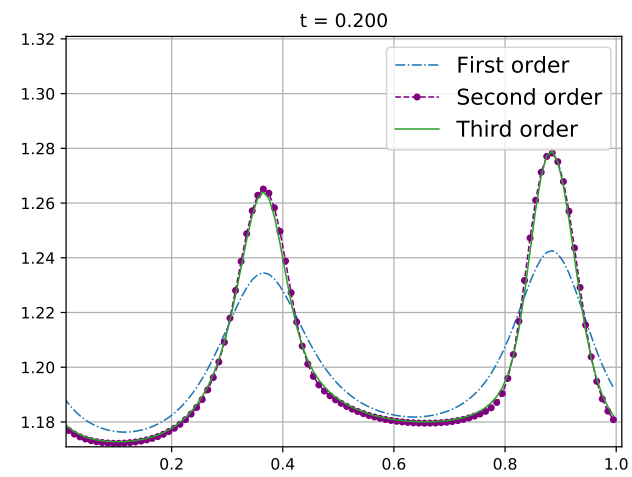

(a) Surface

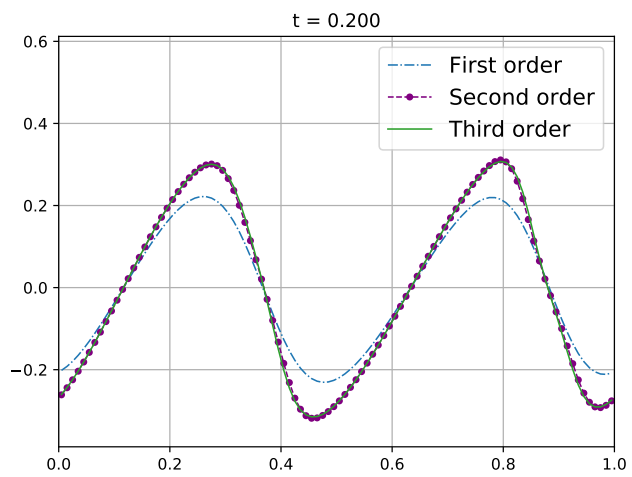

(b) Discharge

Figure 3: Comparison at final times for 100 cell volumes

\begin{tabular}{|c|cc|cc|}
\hline \multirow{2}{*}{ No. of cells } & \multicolumn{2}{|c|}{$h$} & \multicolumn{2}{c|}{$q$} \\
\cline { 2 - 5 } & $L^{1}$ error & order & $L^{1}$ error & order \\
\hline 25 & $1.85 \mathrm{e}-02$ & 0.00 & $1.29 \mathrm{e}-01$ & 0.00 \\
\hline 50 & $1.34 \mathrm{e}-02$ & 0.47 & $8.28 \mathrm{e}-02$ & 0.64 \\
\hline 100 & $8.58 \mathrm{e}-03$ & 0.64 & $4.70 \mathrm{e}-02$ & 0.82 \\
\hline 200 & $5.09 \mathrm{e}-03$ & 0.75 & $2.51 \mathrm{e}-02$ & 0.90 \\
\hline 400 & $2.83 \mathrm{e}-03$ & 0.85 & $1.30 \mathrm{e}-02$ & 0.95 \\
\hline
\end{tabular}

Table 1: $L^{1}$ errors and numerical orders of accuracy: first order scheme.

\begin{tabular}{|c|cc|cc|}
\hline \multirow{2}{*}{ No. of cells } & \multicolumn{2}{|c|}{$h$} & \multicolumn{2}{c|}{$q$} \\
\cline { 2 - 5 } & $L^{1}$ error & order & $L^{1}$ error & order \\
\hline 25 & $1.07 \mathrm{e}-02$ & 0.00 & $4.04 \mathrm{e}-02$ & 0.00 \\
\hline 50 & $4.14 \mathrm{e}-03$ & 1.37 & $1.21 \mathrm{e}-02$ & 1.74 \\
\hline 100 & $1.25 \mathrm{e}-03$ & 1.73 & $3.25 \mathrm{e}-03$ & 1.89 \\
\hline 200 & $3.34 \mathrm{e}-04$ & 1.91 & $8.16 \mathrm{e}-04$ & 1.99 \\
\hline 400 & $8.43 \mathrm{e}-05$ & 1.99 & $2.01 \mathrm{e}-04$ & 2.02 \\
\hline
\end{tabular}

Table 2: $L^{1}$ errors and numerical orders of accuracy: second order scheme.

\begin{tabular}{|c|cc|cc|}
\hline \multirow{2}{*}{ No. of cells } & \multicolumn{2}{|c|}{$h$} & \multicolumn{2}{c|}{$q$} \\
\cline { 2 - 5 } & $L^{1}$ error & order & $L^{1}$ error & order \\
\hline 25 & $6.94 \mathrm{e}-03$ & 0.00 & $1.39 \mathrm{e}-02$ & 0.00 \\
\hline 50 & $1.64 \mathrm{e}-03$ & 2.08 & $3.75 \mathrm{e}-03$ & 1.89 \\
\hline 100 & $2.85 \mathrm{e}-04$ & 2.53 & $8.80 \mathrm{e}-04$ & 2.09 \\
\hline 200 & $4.06 \mathrm{e}-05$ & 2.81 & $1.39 \mathrm{e}-04$ & 2.66 \\
\hline 400 & $5.85 \mathrm{e}-06$ & 2.80 & $2.10 \mathrm{e}-05$ & 2.73 \\
\hline
\end{tabular}

Table 3: $L^{1}$ errors and numerical orders of accuracy: third order scheme. 


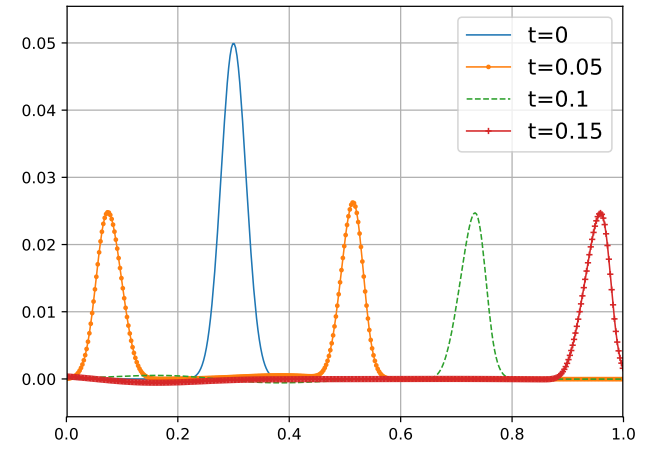

(a) Surface

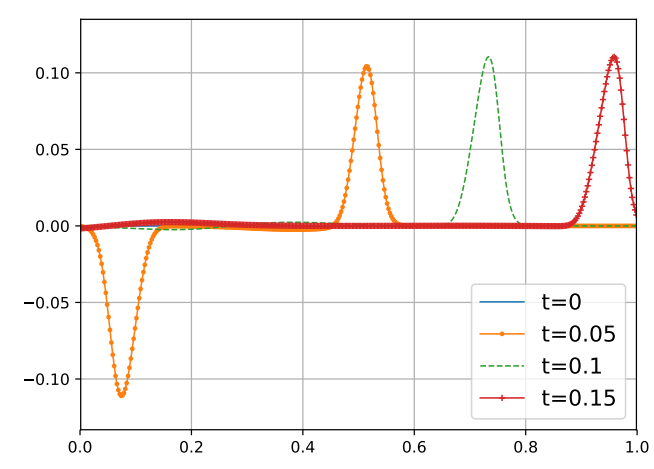

(b) Discharge

Figure 4: Evolution of a perturbation from a lake at rest. Comparison with the reference lake at rest solution.

and we consider a initial small perturbation on the water surface

$$
h(x, t=0)+z(x)=1+0.05 \exp \left(-1000(x-0.3)^{2}\right) .
$$

We simulate this perturbation of the steady state with the third order well-balanced LagrangeProjection scheme using 400 cell volumes. Figure 4 shows the difference of the computed solutions when compared with the unperturbed lake at rest steady state. As expected, the perturbation propagates towards the boundaries until they leave the domain. Afterwards, the unperturbed solution remains. Remark that once the steady state is reached, the scheme preserves it as expected up to an error of order $10^{-13}$.

\subsection{Transcritical flow with shock}

Let us consider now a test from [3]. The space domain is [0,25] and the bottom topography is given by

$$
z(x)= \begin{cases}0.2-0.05(x-10)^{2}, & \text { if } 8<x<12 \\ 0, & \text { otherwise }\end{cases}
$$

the initial data are $h=0.33$ and $q=0.18$, and boundary conditions are $q(x=0)=0.18$ and $h(x=25, t)=0.33$. The final time is $t=200$. Figures 5 and 6 show the comparison between the second order well-balanced Lagrange-Projection scheme with 200 cell points compared with a reference solution with 1000 points using a path-conservative HLL scheme (see [6]). We remark that here we get to a stationary solution which is not smooth. Nevertheless, the results obtained with the LagrangeProjection technique is comparable to the reference solution. Some deviations from the final steady state are obtained as expected, which is specially seen on the discharge and at the shock.

\subsection{Perturbation of a steady state with non-zero velocity}

The objective now is to test the fully well-balanced scheme. Let us consider now a stationary solution with non zero velocity. To do so we set as bottom topography

$$
z(x)=0.5 \exp \left(-200(x-0.5)^{2}\right) ;
$$

and we consider the subcritical stationary solution given by

$$
\left\{\begin{array}{l}
q^{e}(x)=0.5 \\
\frac{\left(q^{e}(x)\right)^{2}}{2\left(h^{e}(x)\right)^{2}}+g\left(h^{e}(x)+z(x)\right)=9.935 .
\end{array}\right.
$$




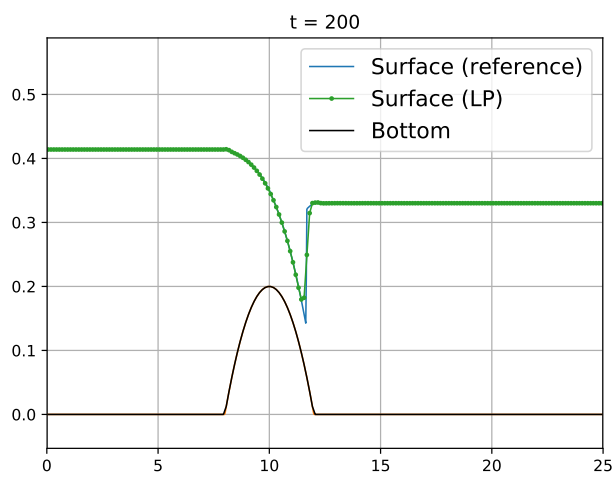

(a) Surface

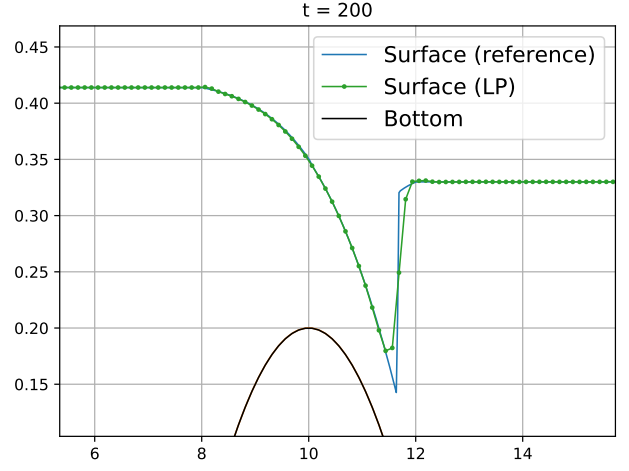

(b) Surface (zoom)

Figure 5: Transcritical flow with shock

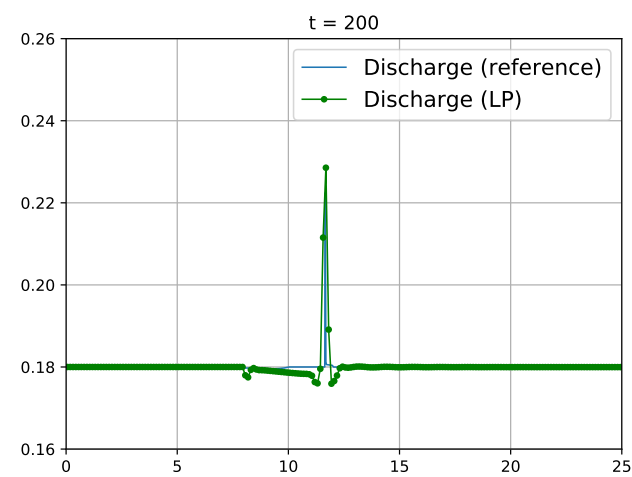

(a) Discharge

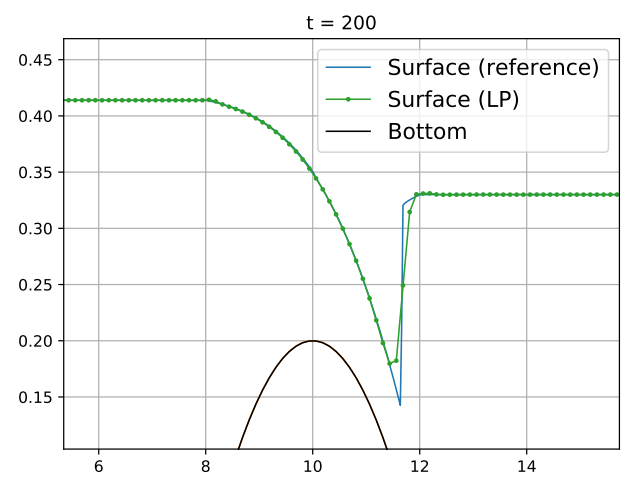

(b) Discharge (zoom)

Figure 6: Transcritical flow with shock 


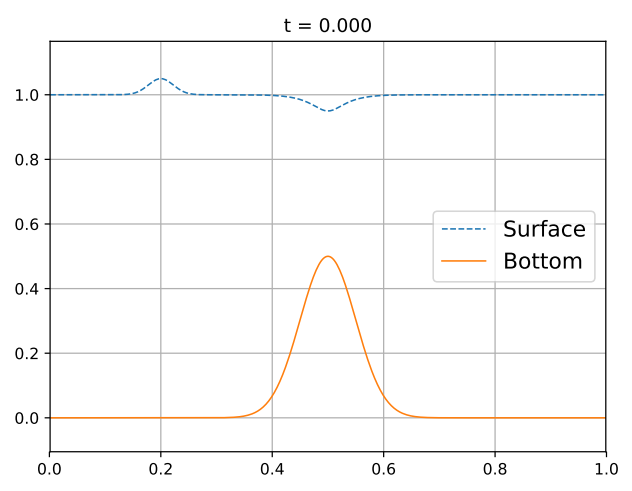

(a) Surface and bottom

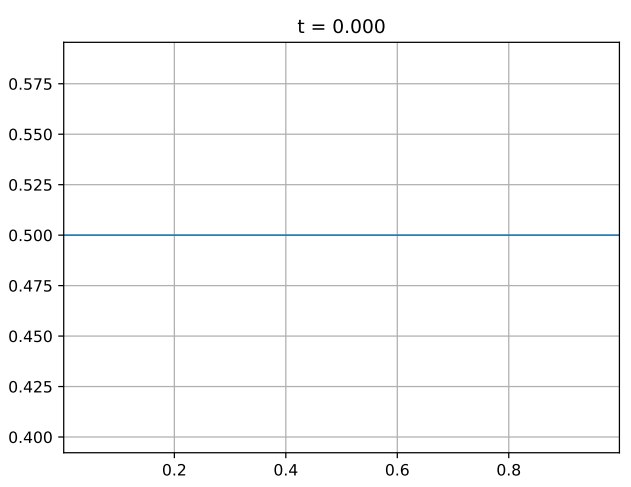

(b) Discharge

Figure 7: Perturbation of a stationary steady state with non-zero velocity: initial condition

The fully-well balanced Lagrange-Projection scheme preserves such a steady state with an error of order $10^{-15}$. We consider now a perturbation on the water thickness of this steady state given by

$$
h(x, t=0)=h^{e}(x)+0.05 \exp \left(-1000(x-0.2)^{2}\right) .
$$

The initial condition is shown in Figure 7.

We solve the system with this initial condition using 400 volume cells on the interval $[0,1]$ using a second order fully well-balanced Lagrange-Projection scheme. The solution is compared with a first order path-conservative HLL scheme, as described in [6], and with a reference solution computed using a very fine grid of 1600 volume cells. The results are shown in Figures 8 and 9. As expected, the scheme preserves exactly the areas where we find the prescribed well-balance solution while solving with accuracy the perturbation.

\section{Conclusions}

We have developed a high-order fully well-balanced approach extension for the Lagrangian-Projection scheme introduced in $[18,10,12,13,9]$. The proposed technique produces good results when applied to shallow water system. Note that, the Lagrange-Projection scheme may be extended to other systems and applications such as bedload sediment transport, turbidity currents, Ripa system, etc. Future works will consider the extension to multi-dimensional domains and high-order semi-implicit schemes.

\section{Acknowledgements}

This research has been partially supported by the Spanish Government and FEDER through the coordinated Research projects MTM 2015-70490-C2-1-R and MTM 2015-70490-C2-2-R as well as the coordinated Research projects RTI2018-096064-B-C21 and RTI2018-096064-B-C22. The authors were partially supported by the Innovative Training Networks (ITN) grant 642768 (ModCompShock). The third author was partially supported by the ANR project ACHYLLES (ANR-14-CE25-0001-03).

The authors are very grateful to S. Del Pino, M. Girardin, P. Hoche, researchers at the CEA, for the interesting discussions just before submitting the present work, and in particular for bringing to their attention the reference [15]. 


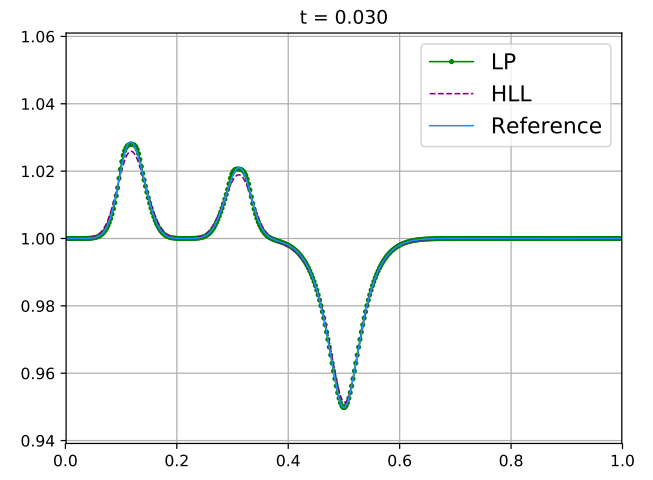

(a) Surface

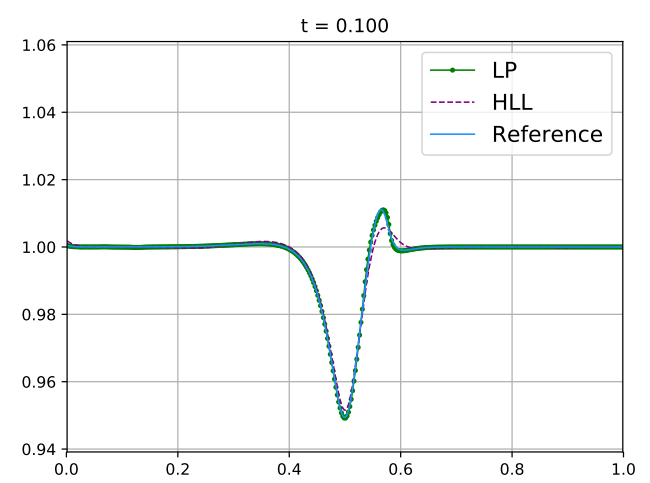

(c) Surface

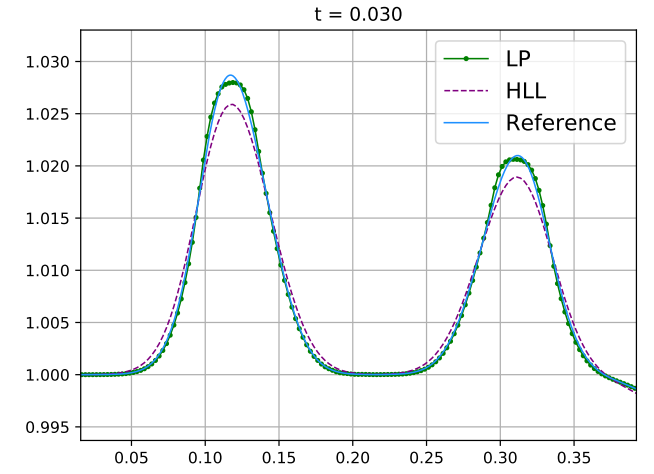

(b) Zoom on the Surface

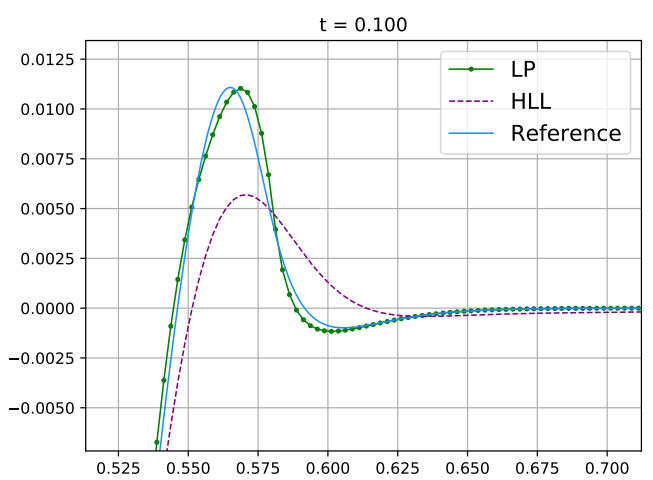

(d) Zoom on the Surface

Figure 8: Perturbation of a stationary steady state with non-zero velocity: Surface evolution 


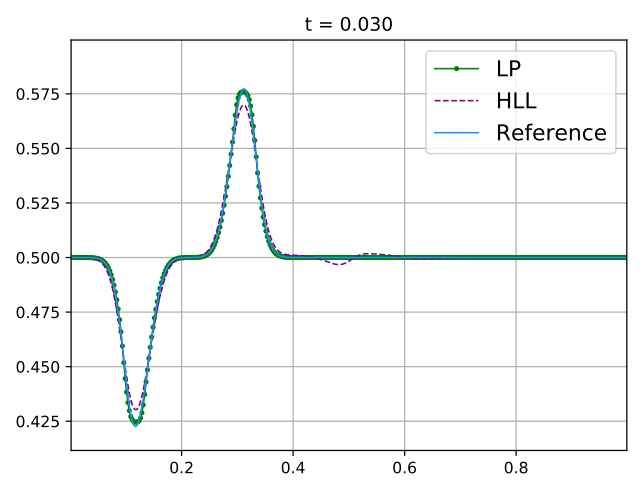

(a) Discharge

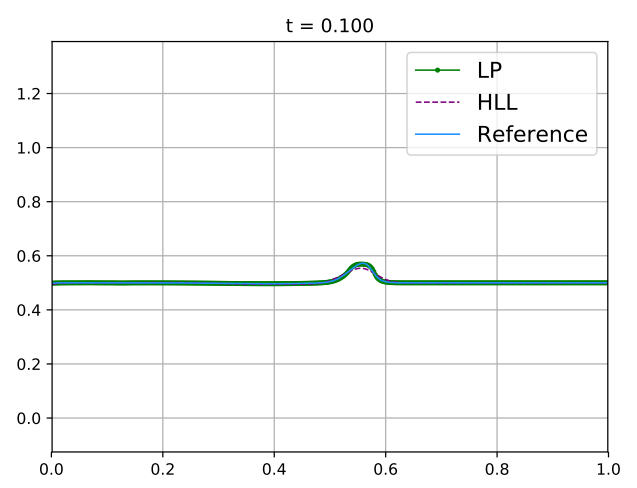

(c) Discharge

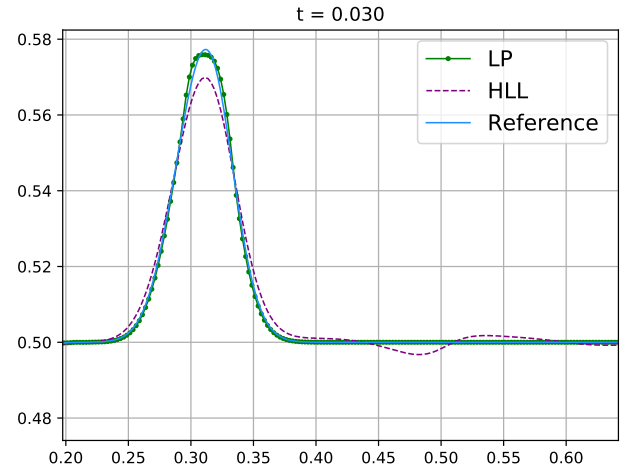

(b) Zoom on the Discharge

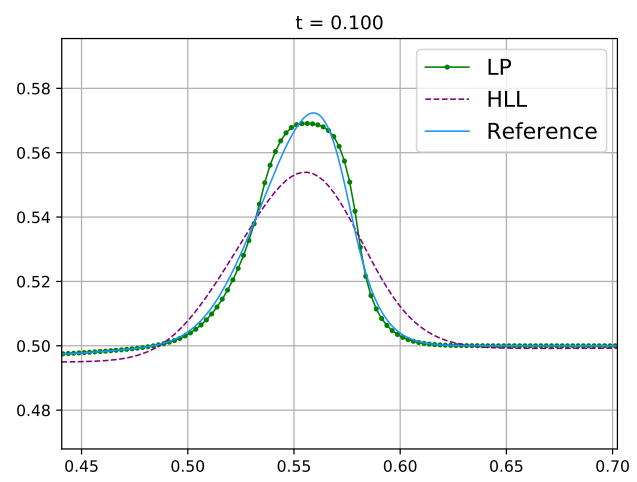

(d) Zoom on the Discharge

Figure 9: Perturbation of a stationary steady state with non-zero velocity: Discharge evolution 


\section{References}

[1] Emmanuel Audusse, Francois Bouchut, Marie-Odile Bristeau, Rupert Klein, and Benoit Perthame. A fast and stable well-balanced scheme with hydrostatic reconstruction for shallow water flows. SIAM Journal on Scientific Computing, 25:2050-2065, 2004.

[2] Christophe Berthon and Christophe Chalons. A fully well-balanced, positive and entropysatisfying Godunov-type method for the shallow-water equations. Mathematics of Computation, 85(299):1281-1307, 2016.

[3] François Bouchut, S. Medvedev, G. Reznik, A. Stegner, and V. Zeitlin. Nonlinear dynamics of rotating shallow water: methods and advances. Edited Series on Advances in Nonlinear Science and Complexity. Elsevier, 2007.

[4] François Bouchut and Tomás Morales de Luna. A subsonic-well-balanced reconstruction scheme for shallow water flows. SIAM J. Numer. Anal., 48(5):1733-1758, 2010.

[5] M. Castro, J. Gallardo, J. López-García, and C. Parés. Well-Balanced High Order Extensions of Godunov's Method for Semilinear Balance Laws. SIAM Journal on Numerical Analysis, 46(2):1012-1039, January 2008.

[6] M. J. Castro, T. Morales de Luna, and C. Parés. Well-Balanced Schemes and Path-Conservative Numerical Methods. In Rémi Abgrall and Chi-Wang Shu, editor, Handbook of Numerical Analysis, volume 18 of Handbook of Numerical Methods for Hyperbolic ProblemsApplied and Modern Issues, pages 131-175. Elsevier, 2017. DOI: 10.1016/bs.hna.2016.10.002.

[7] Manuel J. Castro, Alberto Pardo Milanés, and Carlos Parés. Well-balanced numerical schemes based on a generalized hydrostatic reconstruction technique. Mathematical Models and Methods in Applied Sciences, 17(12):2055, 2007.

[8] M.J. Castro and C. Parés. Well-balanced high-order methods for systems of balance laws. Preprint, 2019.

[9] M. Castro Díaz, C. Chalons, and T. Morales de Luna. A Fully Well-Balanced LagrangeProjection-Type Scheme for the Shallow-Water Equations. SIAM Journal on Numerical Analysis, 56(5):3071-3098, January 2018.

[10] C. Chalons, M. Girardin, and S. Kokh. Large Time Step and Asymptotic Preserving Numerical Schemes for the Gas Dynamics Equations with Source Terms. SIAM Journal on Scientific Computing, 35(6):A2874-A2902, January 2013.

[11] C. Chalons, M. Girardin, and S. Kokh. Operator-splitting based ap schemes for the 1d and 2d gas dynamics equations with stiff sources. AIMS Series on Applied Mathematics, 8:607-614, 2014.

[12] Christophe Chalons, Mathieu Girardin, and Samuel Kokh. An All-Regime Lagrange-Projection Like Scheme for the Gas Dynamics Equations on Unstructured Meshes. Communications in Computational Physics, 20(1):188-233, July 2016.

[13] Christophe Chalons, Pierre Kestener, Samuel Kokh, and Maxime Stauffert. A large time-step and well-balanced Lagrange-projection type scheme for the shallow water equations. Communications in Mathematical Sciences, 15(3):765-788, 2017.

[14] S. Cordier, M.H. Le, and T. Morales de Luna. Bedload transport in shallow water models: Why splitting (may) fail, how hyperbolicity (can) help. Advances in Water Resources, 34(8):980-989, August 2011.

[15] Frédéric Duboc, Cédric Enaux, Stéphane Jaouen, Hervé Jourdren, and Marc Wolff. High-order dimensionally split lagrange-remap schemes for compressible hydrodynamics. Comptes Rendus Mathematique, 348(1):105 - 110, 2010. 
[16] M.J. Castro Díaz, J.A. López-García, and Carlos Parés. High order exactly well-balanced numerical methods for shallow water systems. Journal of Computational Physics, 246:242 - 264, 2013.

[17] F.M. Exner. Über die wechselwirkung zwischen wasser und geschiebe in flüssen. Sitzungsber., Akad. Wissenschaften, pt. IIa:Bd. 134, 1925.

[18] Edwige Godlewski and Pierre-Arnaud Raviart. Numerical approximation of hyperbolic systems of conservation laws, volume 118 of Applied Mathematical Sciences. Springer-Verlag, New York, 1996.

[19] Laurent Gosse. Computing Qualitatively Correct Approximations of Balance Laws: ExponentialFit, Well-Balanced and Asymptotic-Preserving. SEMA SIMAI Springer Series. Springer-Verlag, Mailand, 2013.

[20] Sigal Gottlieb and Chi-Wang Shu. Total variation diminishing Runge-Kutta schemes. Mathematics of Computation, 67(221):73-85, 1998.

[21] Bram van Leer. Towards the ultimate conservative difference scheme. V. A second-order sequel to Godunov's method. Journal of Computational Physics, 32(1):101 - 136, 1979.

[22] Tomás Morales de Luna, Manuel J. Castro Díaz, Carlos Parés Madroñal, and Enrique D. Fernández Nieto. On a shallow water model for the simulation of turbidity currents. Communications in Computational Physics, 6(4):848-882, 2009.

[23] Sebastian Noelle, Yulong Xing, and Chi-Wang Shu. High-order well-balanced finite volume WENO schemes for shallow water equation with moving water. J. Comput. Phys., 226(1):29-58, 2007.

[24] P. Ripa. On improving a one-layer ocean model with thermodynamics. Journal of Fluid Mechanics, 303:169-201, 1995.

[25] G. Russo and A. Khe. High order well-balanced schemes based on numerical reconstruction of the equilibrium variables. In Waves and Stability in Continuous Media, pages 230-241. WORLD SCIENTIFIC, April 2010.

[26] C. Shu. Total-Variation-Diminishing Time Discretizations. SIAM Journal on Scientific and Statistical Computing, 9(6):1073-1084, November 1988.

[27] Chi-Wang Shu. Essentially non-oscillatory and weighted essentially non-oscillatory schemes for hyperbolic conservation laws. In Alfio Quarteroni, editor, Advanced Numerical Approximation of Nonlinear Hyperbolic Equations, number 1697 in Lecture Notes in Mathematics, pages 325-432. Springer Berlin Heidelberg, January 1998.

[28] Yulong Xing, Chi-Wang Shu, and Sebastian Noelle. On the Advantage of Well-Balanced Schemes for Moving-Water Equilibria of the Shallow Water Equations. Journal of Scientific Computing, 48(1):339-349, July 2011.

[29] Xiangxiong Zhang and Chi-Wang Shu. On maximum-principle-satisfying high order schemes for scalar conservation laws. Journal of Computational Physics, 229(9):3091-3120, May 2010. 